\title{
Data set for reporting of ovary, fallopian tube and primary peritoneal carcinoma: recommendations from the International Collaboration on Cancer Reporting (ICCR)
}

W Glenn McCluggage ${ }^{1,15}$, Meagan J Judge ${ }^{2}$, Blaise A Clarke ${ }^{3}$, Ben Davidson ${ }^{4,5}$, C Blake Gilks ${ }^{6}$, Harry Hollema ${ }^{7}$, Jonathan A Ledermann ${ }^{8}$, Xavier Matias-Guiu' ${ }^{9}$, Yoshiki Mikami ${ }^{10}$, Colin JR Stewart ${ }^{11,12}$, Russell Vang ${ }^{13}$ and Lynn Hirschowitz ${ }^{14,16}$

${ }^{1}$ Department of Pathology, Belfast Health and Social Care Trust, Belfast, UK; ${ }^{2}$ Royal College of Pathologists of Australasia, Sydney, NSW, Australia; ${ }^{3}$ Department of Pathology and Laboratory Medicine, University Health Network, University of Toronto, Toronto, ON, Canada; ${ }^{4}$ Department of Pathology, Oslo University Hospital, Norwegian Radium Hospital, Oslo, Norway; ${ }^{5}$ Faculty of Medicine, Institute of Clinical Medicine, University of Oslo, Oslo, Norway; ${ }^{6}$ Department of Pathology, Vancouver General Hospital, University of British Columbia, Vancouver, BC, Canada; ${ }^{7}$ Department of Pathology, University of Groningen, University Medical Center Groningen, Groningen, The Netherlands; ${ }^{8}$ Department of Oncology, UCL Cancer Institute, London, UK; ${ }^{9}$ Department of Pathology and Molecular Genetics and Research Laboratory, Hospital Universitari Arnau de Vilanova, University of Lleida, IRBLleida, Lleida, Spain; ${ }^{10}$ Department of Diagnostic Pathology, Kumamoto University Hospital, Kumamoto, Japan; ${ }^{11}$ Department of Histopathology, King Edward Memorial Hospital, Perth, WA, Australia; ${ }^{12}$ School for Women's and Infant's Health, University of Western Australia, Crawley, WA, Australia; ${ }^{13}$ Department of Pathology (Division of Gynecologic Pathology), The Johns Hopkins University School of Medicine, Baltimore, MD, USA and ${ }^{14}$ Department of Cellular Pathology, Birmingham Women's Hospital, Birmingham, UK

A comprehensive pathological report is essential for optimal patient management, cancer staging and prognostication. In many countries, proforma reports are used but these vary in their content. The International Collaboration on Cancer Reporting (ICCR) is an alliance formed by the Royal College of Pathologists of Australasia, the Royal College of Pathologists of the United Kingdom, the College of American Pathologists, the Canadian Partnership Against Cancer and the European Society of Pathology, with the aim of developing an evidence-based reporting data set for each cancer site. This will reduce the global burden of cancer data set development and reduplication of effort by different international institutions that commission, publish and maintain standardised cancer reporting data sets. The resultant standardisation of cancer reporting will benefit not only those countries directly involved in the collaboration but also others not in a position to develop their own data sets. We describe the development of a cancer data set by the ICCR expert panel for the reporting of primary ovarian, fallopian tube and peritoneal carcinoma and present the 'required' and 'recommended' elements to be included in the report with an explanatory commentary. This data set encompasses the recent International Federation of Obstetricians and Gynaecologists staging system for these neoplasms and the updated World Health Organisation Classification of Tumours of the Female Reproductive Organs. The data set also addresses issues about site assignment of the primary tumour in high-grade serous carcinomas and proposes a scoring system for the assessment of tumour response to neoadjuvant chemotherapy. The widespread implementation of this data set will facilitate consistent and accurate data collection, comparison of epidemiological and pathological parameters between different populations, facilitate research and hopefully will result in improved patient management. Modern Pathology (2015) 28, 1101-1122; doi:10.1038/modpathol.2015.77; published online 19 June 2015

Correspondence: Professor WG McCluggage, FRCPath, Department of Pathology, Royal Group of Hospitals Trust, Grosvenor Road, Belfast BT12 6BA, Northern Ireland.

E-mail: glenn.mccluggage@belfasttrust.hscni.net

${ }^{15}$ Chair of the ICCR Ovarian/Fallopian tube and Primary Peritoneal Site Cancer Panel.

${ }^{16}$ The ICCR Steering Group Representative.

Received 13 January 2015; revised 24 February 2015; accepted 25 February 2015; published online 19 June 2015 
The Colleges of American, Australasian and United Kingdom Pathologists and the Canadian Partnership Against Cancer formed the International Collaboration on Cancer Reporting (ICCR) in 2011 to reduce the global burden of cancer data set development and reduplication of effort by different international institutions that commission, publish and maintain standardised cancer-reporting data sets. Many countries, including those involved in the ICCR, expend a great deal of time, effort and resources to commission, publish and maintain their own standardised cancer-reporting data sets. Other countries lack sufficient pathologist manpower and other resources to develop or implement standardised cancerreporting protocols and will benefit from the availability of internationally accredited data sets.

The ICCR alliance has successfully developed four data sets on prostatic, endometrial and lung cancers and malignant melanoma. All of the data sets are evidence-based, have been produced by a panel of internationally renowned expert pathologists and a single clinician in a specific field, have been subject to international open consultation and are freely available for worldwide use at the following website: http://www.rcpa.edu.au/Library/Practising-Pathology/ ICCR/Cancer-Datasets. The process of production of each of these data sets has been published in peerreviewed journals. ${ }^{1-4}$

The ICCR is currently in the process of becoming incorporated that will facilitate financial support of an expanded membership and allow the continued development of high-quality cancer data sets for international use. The founding members include the original quadripartite group together with the European Society of Pathology. The ICCR has also formed strategic partnerships with the International Agency for Research on Cancer (IARC) (the organisation that is responsible for producing the World Health Organisation (WHO) monographs, the 'Blue books' on tumour classification), the organisations responsible for tumour staging-Union for International Cancer Control (UICC), American Joint Committee on Cancer (AJCC) and the International Federation of Obstetricians and Gynaecologists (FIGO)—and other bodies such as European Organisation for Research and Treatment of Cancer, which develops, conducts, coordinates and promotes translational and clinical research in Europe to improve the management of cancer. These strategic partnerships underpin the cancer data set development process and facilitate the co-ordination of data set development with new tumour classifications (the IARC) and revised cancer staging systems (FIGO, AJCC and UICC).

\section{Materials and methods}

The cancer data sets define the essential pathological data that are required for cancer diagnosis, staging, prognosis and patient management. The aim of standardised data sets is to ensure that histopathology reports include all relevant information (based on current knowledge) and present it in a consistent, concise format that conforms to international standards. Although much of the content of the independently developed cancer reporting protocols is similar, if cancer data are to be aggregated across large populations, much closer harmonisation of approach, uniformity of content and standardised nomenclature to define the data items are needed; the data sets in different parts of the world should include the same items of information, defined in the same way and described using the same terms. Without this, meaningful international comparison, benchmarking and epidemiological analysis are not possible.

The ICCR has developed and ratified a suite of standard operating procedures for the process of data set development (described in earlier publications ${ }^{1-4}$ ) and also defined the selection process, roles and responsibilities of the chair, expert panel members, the ICCR Steering Committee representative on the panel and the project manager.

The scope of this data set was to cover resection specimens of primary borderline and malignant epithelial tumours of the ovary, fallopian tube and peritoneum. After the expert panel was established, the project manager collated existing international data sets for these tumours and scheduled a series of teleconferences to review and discuss each of the elements in the collated data set. As FIGO staging of ovarian, fallopian tube and primary peritoneal carcinoma has now been revised and amalgamated into one unified staging system, ${ }^{5}$ the Chair of the expert panel (WGM) took the decision to produce a single cancer data set for these tumours.

The elements under discussion by the expert panel included REQUIRED elements, defined as those which are unanimously agreed by the panel to be essential for the histological diagnosis, clinical management, staging or prognosis of ovarian, fallopian tube and primary peritoneal cancer, and RECOMMENDED elements, defined as non-mandatory which are clinically important and recommended as good practice and should ideally be included in the data set but which are not yet validated or regularly used in patient management. Evidentiary support at Level III-2 or above (based on prognostic factors in the NHMRC levels of evidence document and defined as 'Analysis of prognostic factors amongst persons in a single arm of a randomised controlled trial' $)^{6}$ is required to support required (mandatory) elements. Rarely, where Level III-2 evidence is not available, an element can be categorised as required with unanimous agreement of the expert panel. Required elements are mandatory and the sum of these is the minimum information that should be included on the pathology report. In many countries, most or all of the recommended elements will also be included on the pathology report.

Once the elements were agreed by the panel, the next phase of the data set development process was to propose the value list or response to each element, 
e.g., 'present', 'not assessable', 'not indicated', to avoid any ambiguity in the assessment or meaning of the element. The Chair of the expert panel then assigned the writing of a commentary for each data item, based on a review of the current literature, to different members of the panel. The commentary comprises explanatory text, diagrams or tables to: (i) clarify required and recommended elements, (ii) explain why each element is necessary (e.g., how does it assist with clinical management or prognosis of the specific cancer); and (iii) define the way each element should be reported. The required elements and associated commentaries are presented below followed by the recommended elements and commentaries.

\section{Required Data Elements \\ Prior chemotherapy}

Table 1 lists the required data elements, preoperative chemotherapy may significantly alter the gross and microscopic appearance of the tumour and result in difficulties in tumour typing and grading and tumour down-staging. In some cases, there may be no residual tumour. If neoadjuvant chemotherapy is being administered, a pretreatment tissue biopsy should be obtained and used for tumour typing and grading. If this is not possible, then the diagnosis of malignancy can be made on cytological examination of ascitic fluid, preferably with immunohistochemistry performed on a cell block preparation; however, this should only be in exceptional circumstances. Markers of value in tumour typing are discussed later (section on 'Immunohistochemical markers').

\section{Specimen type}

Providing information about the specimen type (organs and tissues received) is regarded as an integral part of the reporting of ovarian, tubal and primary peritoneal cancers. Although the nature of the specimen/s submitted for pathological assessment may be deduced from the surgical procedure, specifying the nature of specimen received provides complementary information and confirmation that entire organ/s have been resected and submitted.

\section{Specimen integrity}

Assessment of the integrity of the specimen (ovary or tube) is important, particularly for substaging of organ-confined disease (stage I). Information should include whether the ovarian capsule or tubal serosa is intact or ruptured and also whether there is tumour on the surface or whether the tumour was received fragmented or intact. In case of capsule rupture, it is recommended to try to ascertain whether rupture occurred before or during surgery (this is important in substaging FIGO stage IC disease - see next paragraph), although obviously this information should be provided by the surgeon. Occasionally, there is microscopic ovarian surface involvement in the absence of gross capsular deficiency and this should be recorded (section on 'Histological sites of tumour involvement').

Approximately $25 \%$ of ovarian cancers are FIGO stage I at diagnosis, with a 5-year-survival of 83-90\%.7,8 According to the 2014 FIGO staging system for ovarian, tubal and primary peritoneal cancer, ${ }^{5}$ ovarian capsular or tubal serosal rupture before surgery is considered stage IC2 while intra-operative rupture is $1 \mathrm{C} 1$. There is some controversy as to whether rupture during surgery worsens the prognosis in the absence of surface excrescences, ascites or positive washings. Some studies showed a higher risk of recurrence in association with intra-operative ovarian capsular rupture, ${ }^{9,10}$ whereas others did not. ${ }^{11-13}$

A recent meta-analysis ${ }^{5}$ assessed the impact of intra-operative rupture on prognosis, after analysing 9 eligible studies which included 2382 patients. Patients with preoperative capsular rupture showed poorer progression-free survival (PFS) than those with no rupture or intra-operative rupture. In the subanalyses, preoperative rupture was associated

Table 1 Required data items for pathological reporting of ovarian, fallopian tube and primary peritoneal carcinomas

\begin{tabular}{|c|c|c|}
\hline Clinical & Macroscopic & Microscopic \\
\hline Prior chemotherapy & Specimen integrity & Histological tumour type (for carcinomas and borderline tumours) \\
\hline \multirow[t]{12}{*}{ Specimen type } & Macroscopic tumour site & $\begin{array}{l}\text { Tumour grade (for serous, endometrioid, clear cell and undifferentiated } \\
\text { carcinoma and carcinosarcoma) }\end{array}$ \\
\hline & Macroscopic description of omentum: & Borderline tumours: \\
\hline & Omental dimensions & Histological tumour type \\
\hline & Omental involvement & Special features: \\
\hline & Maximum dimension of the largest & Micropapillary architecture for serous borderline tumour \\
\hline & deposit & Microinvasion \\
\hline & & Implants for serous and seromucinous borderline tumour \\
\hline & & Serous tubal intraepithelial carcinoma (STIC) \\
\hline & & Histological sites of tumour involvement \\
\hline & & Peritoneal cytology \\
\hline & & Lymph node status \\
\hline & & Provisional Pathological Staging Pre-MDTM (FIGO) \\
\hline
\end{tabular}

${ }^{\mathrm{a}}$ Clear cell, undifferentiated and carcinosarcoma are automatically high grade (see text). 
with a worse prognosis, and intra-operative rupture had a poorer PFS than no rupture. However, no difference in PFS was found between intra-operative rupture and no rupture in patients who underwent a complete surgical staging operation, with or without adjuvant platinum-based chemotherapy.

There is some evidence to suggest that clear cell carcinomas exhibit a higher risk of rupture, ${ }^{14}$ probably related to adhesions to the surrounding tissues, associated with tumour invasion or endometriosis. ${ }^{15}$ Capsular rupture has also been associated with pregnancy. ${ }^{16}$

\section{Histological sites of tumour involvement}

Histological sites of tumour involvement should be recorded as this is necessary for tumour staging.

Although site assignment (tube vs ovary vs peritoneum) for clear cell, endometrioid, low-grade serous and mucinous carcinomas is generally not problematic, the same is not true for high-grade serous carcinomas (HGSCs).

It was first recognised in 2001 (refs. 17,18) that a high percentage of so-called ovarian HGSC in women with germline BRCA1 mutations arise in the fimbrial end of the fallopian tube. This was first noticed in risk-reducing salpingo-oophorectomy specimens where early, preinvasive, HGSCs are much more likely to be present in the fallopian tube than ovary. These serous tubal intraepithelial carcinomas (STICs) harbour identical p53 mutations to the extratubal tumour, establishing that they are clonal. ${ }^{19}$ Comparison of telomere length and centrosome amplification in matched STIC and ovarian HGSC suggests that the STICs develop before the ovarian tumours. ${ }^{20,21}$ Finally, although numbers are small, early, incidental non-BRCA1/2-associated (sporadic) HGSCs are predominantly detected in the fallopian tube mucosa, especially the fimbriae, rather than the ovary. ${ }^{22}$ In summary, there is compelling evidence that in a significant majority of cases, but possibly not all, the precursors of HGSC originate in the fallopian tube in patients with germline BRCA1 mutations, and accumulating evidence suggest that this is also true for sporadic HGSC. Assignment of primary site should therefore reflect our current understanding of where HGSCs originate, based on data from the study of early incidental or preinvasive HGSC. It is also relevant that some cases of ovarian and primary peritoneal HGSCs do not show STIC lesions despite complete examination of the fallopian tube. In a consecutive series of non-uterine HGSCs classified as ovarian or peritoneal based on pre-FIGO 2014 criteria in which the fallopian tubes were examined in their entirety, STICs were identified in 59\% of cases and invasive HGSC of the mucosa of the fallopian tube in an additional 15\% of cases. $^{23}$ In other cases, the fimbrial end of the fallopian tube was obliterated by a tubo-ovarian mass.

According to the FIGO 2014 staging system, the primary site of non-uterine HGSC is designated as ovarian, tubal or primary peritoneal. ${ }^{5}$ In some cases, it may not be possible to ascertain the primary site of origin, and these should be categorised as 'undesignated' in the new staging system. ${ }^{5}$ The descriptor 'tubo-ovarian HGSC' can also be used in practice for those cases of advanced stage HGSC where there is uncertainty about primary site. The problems in ascertaining the primary site and the variation in practice among pathologists have significant implications for epidemiological studies, determination of tumour incidence and mortality, data collection by cancer registries and entry into clinical trials. Based on a recent publication, recommendations for assigning the site of origin of extra-uterine HGSC are provided in the following section. ${ }^{24}$ Using these criteria, assignment of primary site is no longer based on the site of the greatest volume/size of tumour, but in the presence of STIC or invasive HGSC in the tubal mucosa, a fallopian tube origin is rendered, although as discussed later (section on 'Serous tubal intraepithelial carcinoma'), fallopian tube mucosal involvement by uterine or non-gynaecological primary tumours can occur and mimic STIC. Application of these criteria will be important in ensuring consistency between different pathologists in assigning the site of origin of HGSC with obvious important implications for cancer registration and other parameters.

Suggestions for assigning the site of origin. The following suggestions are not intended to be an exhaustive list nor are they intended to be binding, and assignment of origin in an individual case is left to the discretion of the pathologist and the clinical team, ideally in the setting of a multidisciplinary team meeting (MDTM). Undoubtedly, there will be evolution over time in our ability to accurately assign the primary tumour site but the following are intended as practical guidelines for handling cases at the present time (Figure 1). ${ }^{24}$

(1) The fallopian tubes, or at least their fimbrial ends, should be totally sampled in all the cases of HGSC by a SEE-FIM-like protocol ${ }^{19}$ to avoid missing this important site of disease, which probably represents the tumour origin in the majority of cases.

(2) The presence of STIC, in the absence of invasive disease in the fallopian tube, should be considered as tubal involvement for staging purposes.

(3) The presence of STIC without invasion or extratubal spread should be staged as FIGO stage IA tubal carcinoma (although these have a favourable prognosis, based on limited experience to date ${ }^{25}$ ) but with an annotation that there is no invasive carcinoma.

(4) Cases with only STIC, ovarian surface involvement or parenchymal involvement not exceeding $5 \mathrm{~mm}$ and widespread peritoneal involvement, which would traditionally be categorised as primary peritoneal carcinoma, ${ }^{26}$ should be classified as tubal primaries. 


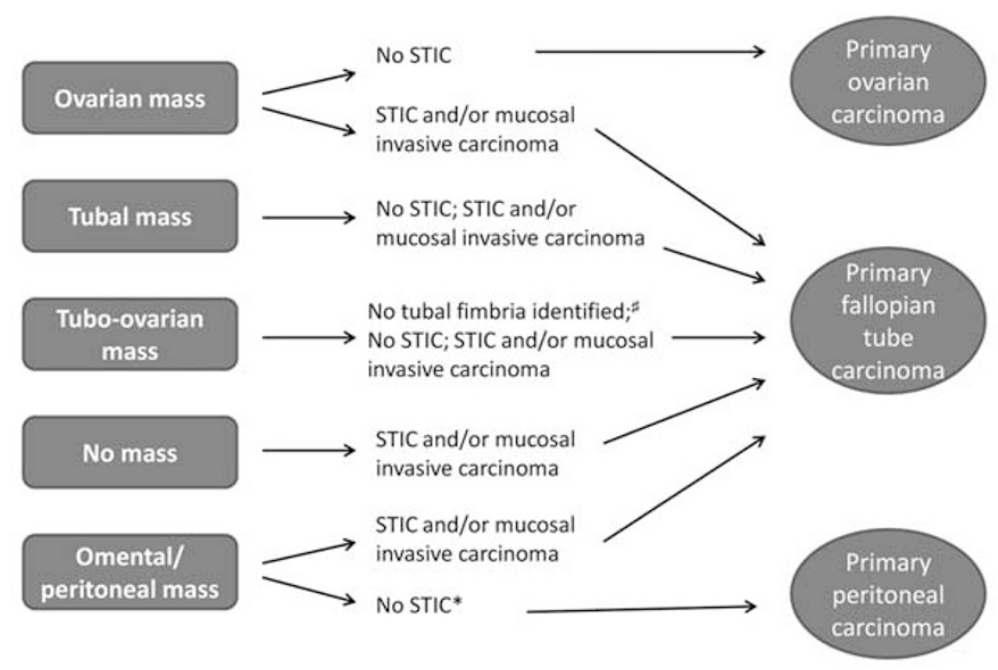

\# Failure to detect the tubal fimbria implies overgrowth by tumour

* Apply criteria as specified in Required Data Elements (section iv: Tumour site).

Figure 1 Flowchart for assigning the site of origin of extrauterine high-grade serous carcinoma.

(5) Cases with invasive HGSC located within the mucosa of the fallopian tube, including its fimbrial end, with or without STIC in any portion of the fallopian tube and with no, minimal or even substantial ovarian involvement should be categorised as tubal primaries.

(6) Cases in which the fallopian tube is not identifiable, having presumably been overgrown by the ipsilateral adnexal mass, or the distal end of the fallopian tube is incorporated into a large tubo-ovarian mass should also, based on current understanding, be diagnosed as tubal primaries. It is emphasised that a careful effort must be made to identify the tube in all the cases.

(7) Cases with a dominant ovarian mass(es) and identifiable fallopian tubes with STIC should be classified as tubal primaries.

(8) Cases with a dominant ovarian mass(es) and identifiable fallopian tubes without STIC should be classified as ovarian primaries.

(9) Cases should be categorised as primary peritoneal carcinoma by the conventional criteria below $^{26}$ and only after complete examination of the fallopian tubes (including the nonfimbrial portions) has excluded the presence of STIC or a small tubal HGSC

- both ovaries must be normal in size or enlarged by a benign process

- the involvement in the extra-ovarian sites must be greater than the involvement on the surface of either ovary

- the ovarian tumour involvement must be nonexistent, confined to the ovarian surface without stromal invasion or involve the cortical stroma with tumour size $<5 \times 5 \mathrm{~mm}^{2}$.
(10) All cases classified as 'undesignated' for FIGO staging purposes should be further described as 'tubo-ovarian' or 'tubal/ovarian' to distinguish them from serous carcinoma originating in the uterus. Using the suggestions presented here, these should represent a small proportion of HGSC.

(11) Cases with unilateral or bilateral HGSC in the ovary and/or STIC or HGSC in the tube but with an endometrial serous intraepithelial or invasive carcinoma should be carefully evaluated for an endometrial vs a tubo-ovarian primary (WT1 may be of value in such cases; see section below on 'Immunohistochemical markers: Distinction between ovarian and uterine carcinoma'); a majority of such cases will represent adnexal metastases from an endometrial serous carcinoma.

\section{Macroscopic description of omentum}

Three dimensions of the omentum should be provided in the pathology report to document the size of the specimen received for pathological examination. This may be useful in certain scenarios to direct the need for further surgery. For example, if initially only an omental biopsy was performed, further surgery may be undertaken to remove the remainder of the omentum. The size of the specimen is also helpful to determine the extent of sampling for histological examination. No standardised guidelines have been developed for sampling omental specimens in cases of ovarian carcinoma or borderline tumours. However, in the setting of a grossly involved omentum, submitting one block for histological examination is probably sufficient. ${ }^{27,28}$ In patients who have received neoadjuvant chemo- 
therapy, where histological assessment of tumour response to therapy is recommended (see recommended data items below), examination of 4-6 blocks of omentum is suggested. For grossly negative omental specimens, the sampling recommendations are variable-sampling of $3-5$ blocks is recommended in one study, ${ }^{28}$ other studies suggest 1 block for every $67 \mathrm{~mm}$ of maximal dimension of omentum ${ }^{27}$ or at least 1 block for every $20 \mathrm{~mm}$ of maximum omental dimension. ${ }^{29}$ Taking $4-6$ blocks in cases where the omentum is grossly negative in patients with an ovarian carcinoma or borderline tumour is recommended.

The size of the largest tumour deposit should be recorded in the pathology report. This is critical for determining the pathological stage. Microscopic tumour, which is not grossly evident, macroscopically evident tumour $\leq 20 \mathrm{~mm}$ and macroscopically evident tumour $>20 \mathrm{~mm}$ correspond to FIGO stages IIIA2, IIIB and IIIC, respectively (FIGO 2014). ${ }^{5}$

\section{Histological tumour type}

All ovarian epithelial malignancies and borderline tumours should be typed according to the WHO classification (Tables 2-4). ${ }^{30}$ There are five major subtypes of primary ovarian carcinoma, high-grade serous, clear cell, endometrioid, mucinous and lowgrade serous. ${ }^{31-34}$ There are also other uncommon minor subtypes, those listed by the WHO, including malignant Brenner tumour, seromucinous carcinoma and undifferentiated carcinoma. ${ }^{30}$ Carcinosarcoma is a mixed epithelial and mesenchymal malignancy but is included in the category of epithelial malignancies in this data set as most are of epithelial origin and histogenesis. ${ }^{35}$

Although management of ovarian carcinoma is, at present, largely dependent on tumour stage and grade, accurate typing will almost certainly become more important in the future with the introduction of targeted therapies and specific treatments for different tumour types. This is in part because, although clinically often considered as one disease, there is an increasing realisation that the different morphological subtypes of ovarian carcinoma have a different pathogenesis, are associated with distinct molecular alterations and have a different natural history, response to traditional chemotherapy and prognosis. ${ }^{31-34}$ Tumour typing may also be important in identifying or initiating testing for an underlying genetic predisposition; for example, HGSC may be associated with the underlying BRCA1/2 mutation while endometrioid and clear cell carcinomas can occur in patients with Lynch syndrome (LS). ${ }^{36}$ The most common ovarian carcinoma is HGSC (approximately 70\%) followed by clear cell and endometrioid. ${ }^{37,38}$ Mucinous and low-grade serous are less common. Approximately $90 \%$ of the advanced stage ovarian carcinomas (stage III/IV) are high-grade serous in type. ${ }^{37,38}$

Most primary tubal carcinomas are high-grade serous or endometrioid and most primary peritoneal
Table 2 WHO classification of primary ovarian epithelial tumours

Serous tumours

Borderline

Serous borderline tumour /atypical proliferative serous tumour

Serous borderline tumour-micropapillary variant/ non-invasive low-grade serous carcinoma

Malignant

Low-grade serous carcinoma

High-grade serous carcinoma

Mucinous tumours

Borderline

Mucinous borderline tumour/Atypical proliferative mucinous tumour

Malignant

Mucinous carcinoma

Endometrioid tumours

Borderline

Endometrioid borderline tumour/Atypical proliferative endometrioid tumour

Malignant

Endometrioid carcinoma

Clear cell tumours

Borderline

Clear cell borderline tumour/atypical proliferative clear cell tumour

Malignant

Clear cell carcinoma

Brenner tumours

Borderline

Borderline Brenner tumour/atypical proliferative Brenner tumour

Malignant

Malignant Brenner tumour

Seromucinous tumours

Borderline

Seromucinous borderline tumour/atypical proliferative seromucinous tumour

Malignant

Seromucinous carcinoma

Undifferentiated carcinoma

Mixed epithelial and mesenchymal tumours Carcinosarcoma

Note: a code for mixed cell adenocarcinoma is not included in the above list, but the code M8323/3 is recommended if this diagnosis is made.

carcinomas are of high-grade serous type. As discussed in the sections on tumour site, it may be difficult to ascertain the origin of a HGSC as multiple sites are often involved.

Mixed ovarian carcinomas are now considered to be uncommon. The current 2014 WHO classification does not include a category of mixed carcinoma, ${ }^{31}$ but the prior classification stated that a diagnosis of mixed carcinoma should only be made if the minor component represents $>10 \%$ of the neoplasm. ${ }^{31}$ However, it is recommended that all different 
Table 3 WHO classification of primary epithelial tumours of the fallopian tube

\begin{tabular}{l} 
Epithelial precursor lesion \\
\hline Serous tubal intraepithelial carcinoma \\
Epithelial borderline tumour \\
Serous borderline tumour/atypical proliferative serous tumour \\
Malignant epithelial tumours \\
Low-grade serous carcinoma \\
High-grade serous carcinoma \\
Endometrioid carcinoma \\
Undifferentiated carcinoma \\
Mucinous carcinoma \\
Transitional cell carcinoma \\
Clear cell carcinoma \\
Mixed epithelial-mesenchymal tumours \\
Carcinosarcoma
\end{tabular}

Note: a code for mixed cell adenocarcinoma is not included in the above list, but the code M8323/3 is recommended if this diagnosis is made.

Table 4 WHO classification of primary peritoneal tumours

Epithelial tumours of Müllerian type

Serous borderline tumour/atypical proliferative serous tumour Low-grade serous carcinoma

High-grade serous carcinoma

Others

Note: a code for mixed cell adenocarcinoma is not included in the above list, but the code M8323/3 is recommended if this diagnosis is made.

morphological subtypes in an ovarian carcinoma are documented, even if they comprise $<10 \%$ of the neoplasm. As stated, mixed carcinomas in the ovary are uncommon, the most prevalent combination being clear cell and endometrioid (both of these tumour types often arise in endometriosis). Most neoplasms that were previously classified as mixed serous and endometrioid and mixed serous and clear cell represent HGSCs with pseudoendometrioid areas and areas of cytoplasmic clearing, respectively. In such cases, immunohistochemical markers, especially WT1, may be useful (see section on 'Immunohistochemical markers' below).

Borderline tumours should also be typed according to WHO criteria. The most common subtypes are serous and mucinous (intestinal type). Seromucinous, endometrioid, clear cell and Brenner subtypes also occur.

\section{Tumour grade}

Assessment of histological grade is important for patient management and prognosis and is a required element. ${ }^{39}$ Although some universal grading systems, for example, the Shimizu-Silverberg system, ${ }^{40}$ are in use that are applicable to all ovarian epithelial malignancies, the ICCR recommends that different grading systems should be used for the different morphological subtypes. If chemotherapy has been administered, tumour grading (and typing) may need to be based on the prechemotherapy biopsy.

Serous carcinoma. Improvements in the understanding of the natural history and molecular pathology of serous carcinoma have demonstrated that HGSC and low-grade serous carcinoma are different tumour types with a different underlying pathogenesis and associated with different molecular events and prognosis. ${ }^{40-43}$ Serous carcinomas are now classified as low-grade or high-grade and this has been endorsed by WHO 2014, ${ }^{30}$ with the recognition that these are two different tumour types rather than low-grade and high-grade variants of the same tumour type.

Clear cell and undifferentiated carcinomas and carcinosarcomas. These are high-grade tumours by definition. Although some publications suggest that clear cell carcinomas should be graded according to a three-tier system, ${ }^{44}$ there is no consensus about this.

Endometrioid carcinoma. Grading of endometrioid carcinomas is identical to that of uterine endometrioid carcinomas ${ }^{45-50}$ and is of prognostic and therapeutic significance. A significant majority of ovarian endometrioid carcinomas is of grades 1 and 2. However, there is a subset of grade 3 endometrioid carcinomas that should be diagnosed with caution, as a significant proportion of such tumours are in fact HGSCs with a glandular growth pattern. Immunohistochemistry is useful in this regard (see section on 'Immunohistochemical markers' below). The 1988 FIGO grading system is widely used for grading endometrioid carcinomas and is recommended by the ICCR. The FIGO system is based on architecture; tumours with $<5 \%$ solid glandular component are grade 1 , those with $5-50 \%$ solid areas are grade 2 and tumours with $>50 \%$ of solid glandular component are classified as grade 3 . When grades 1 and 2 tumours show notable nuclear atypia, the histological grade is increased by one.

There are no published recommendations for the grading of seromucinous carcinomas and malignant Brenner tumours, two rare ovarian malignancies, which are included in the recent WHO Classification and for which no grading recommendations have been provided. ${ }^{30}$ As seromucinous carcinomas have some features in common with endometrioid carcinomas, the ICCR recommends that they should be graded in the same way as endometrioid ovarian carcinomas, i.e., according to the 1988 FIGO grading system. $^{45}$ 


\section{Borderline tumours}

Histological type. Terminology for ovarian borderline tumours has evolved over several years. ${ }^{29,51}$ The preferred terminology is borderline tumour, for example, serous or mucinous borderline tumour, and this has been endorsed in the 2014 WHO Classification. ${ }^{30} \mathrm{An}$ acceptable synonym is atypical proliferative tumour. ${ }^{30}$ Serous borderline tumours, which have been previously designated typical and micropapillary types, are now classified as serous borderline tumour/atypical proliferative serous tumour and micropapillary variant of serous borderline tumour/non-invasive low-grade serous carcinoma, respectively, in the 2014 WHO Classification for gynaecological tumours. ${ }^{30,52}$ For mucinous, endometrioid, clear cell, Brenner and seromucinous tumours, borderline tumour/atypical proliferative tumour terminology is also used in the 2014 WHO Classification. ${ }^{30,53-57}$ The term low malignant potential is not recommended. ${ }^{30,52-57}$ Synonyms for seromucinous tumours include endocervical-type mucinous borderline tumour, Müllerian mucinous borderline tumour and atypical proliferative (borderline) Müllerian tumour. ${ }^{56}$

Determining the lowest threshold for the diagnosis of a borderline tumour in the setting of a cystadenoma/cystadenofibroma with minimal epithelial proliferation can be subjective and quantitative criteria have been suggested: cystadenomas/cystadenofibromas with qualitatively sufficient epithelial stratification/ complexity involving $\geq 10 \%$ of the epithelial volume are designated as borderline tumours arising within a cystadenoma/cystadenofibroma. ${ }^{29,52,57}$ However, many would still diagnose a borderline tumour in which the epithelial stratification/complexity involves $<10 \%$ of the epithelial volume.

Special features. Micropapillary architecture: As serous borderline tumour/atypical proliferative serous tumour can exhibit variable degrees of micropapillary architecture, a diagnosis of micropapillary variant of serous borderline tumour is based on the presence of $\geq 5 \mathrm{~mm}$ of confluent micropapillary growth. ${ }^{30,52}$

Microinvasion: A standardised quantitative criterion for distinguishing microinvasion from frankly invasive carcinoma within a borderline tumour has not been established, and varying definitions have been used in different studies, including $1 \mathrm{~mm}$, $2 \mathrm{~mm}, 3 \mathrm{~mm}, 5 \mathrm{~mm}$ and $10 \mathrm{~mm}^{2}$ as the upper limits of microinvasion. ${ }^{29,51,52,57,58}$ The 2014 WHO Classification suggests a cutoff of $5 \mathrm{~mm} .{ }^{30}$ Some groups distinguish two patterns of stromal invasion in serous tumours that quantitatively falls short of frankly invasive carcinoma ( $<5 \mathrm{~mm})$ —conventional 'microinvasion' (isolated and/or small clusters of eosinophilic cells) and 'microinvasive carcinoma' (glandular or micropapillary patterns qualitatively analogous to low-grade serous carcinoma). ${ }^{29,51,52}$ However, other investigators do not advocate this distinction. Because of insufficient numbers of cases in the literature, definitive conclusions regarding the clinical significance of this distinction cannot be drawn. ${ }^{51,52,59}$ Analogous to the situation for serous tumours, some investigators advocate the separation of 'microinvasion' from 'microinvasive carcinoma' in mucinous borderline tumours while others use these two terms synonymously. ${ }^{57,58}$

Implants: Extra-ovarian implants occur in approximately $20 \%$ of serous borderline tumours and are more common with exophytic neoplasms. The most important adverse prognostic factor for serous borderline tumours is the presence of invasive implants in extra-ovarian tissues, with non-invasive implants having a favourable prognosis. Specifying the location and size of implants is important for determining the FIGO stage. $^{5}$ Non-invasive and invasive implants may co-exist in the same specimen. Non-invasive implants are subclassified as epithelial or desmoplastic types. ${ }^{29}$ Epithelial-type non-invasive implants resemble detached fragments of a serous borderline tumour involving extraovarian tissues. They do not exhibit infiltration of underlying tissue, and they are often present within mesothelial or epithelial-lined spaces although they may be adherent to the serosal surface. Desmoplastic non-invasive implants are composed of glands or papillary clusters within a predominant fibroblastic or granulation tissue-like stroma, but they do not exhibit infiltration of adjacent tissue. Often these are located on serosal surfaces or within septa in the omentum. Note that the presence of isolated individual or small clusters of eosinophilic epithelial cells within the stroma is generally considered to be within the spectrum of desmoplastic non-invasive implants rather than representing an invasive implant. ${ }^{51,52}$

The most widely used criterion for diagnosing invasive implants is destructive invasion of the underlying tissue. ${ }^{60}$ Invasive implants often feature markedly crowded epithelial nests, glands or micropapillary clusters with a haphazard arrangement. The nests, glands and papillae are sometimes surrounded by clefts. As some peritoneal staging biopsies may be superficial without sufficient underlying tissue to assess invasion, expanded criteria for invasive implants have been proposed for cases without classic patterns of invasion. ${ }^{61}$ These criteria include micropapillary architecture resembling micropapillary serous borderline tumour and clusters of tumour within clear lacunar spaces. Not all gynaecological pathologists accept these expanded criteria, ${ }^{29,51}$ but they have been shown to correlate with poor outcome. ${ }^{61} \mathrm{We}$ feel this is an area where further studies are needed to clarify the prognostic significance of these expanded criteria.

In occasional cases, it may not be possible to definitively distinguish non-invasive from invasive implants and the recommendation is to designate such implants as being of indeterminate type. ${ }^{62}$ This terminology should only be used sparingly, and obtaining a specialist gynaecological pathology opinion and submitting additional sections for histo- 
logical examination (if an omentectomy specimen) may be useful.

When diagnosing invasive implants, the report should state that these represent extra-ovarian lowgrade serous carcinoma; this has been endorsed in the 2014 WHO blue book. ${ }^{29,30,51,52,61}$ It is unclear whether invasive implants involving extra-ovarian sites in association with an ovarian serous borderline tumour represent metastases from the serous borderline tumour or an independent primary peritoneal tumour. A number of molecular studies analysing primary ovarian tumours with their associated implants have yielded varying results, ${ }^{51}$ but a recent study of a large population-based cohort has shown that the vast majority of implants are clonally related to the primary ovarian tumour. ${ }^{63}$ Most of the cases from that study were non-invasive implants; however, all 10 invasive implants had the same mutational status (KRAS mutation, BRAF mutation or wild-type $K R A S / B R A F$ ) as the corresponding serous borderline tumour, suggesting that invasive implants are clonally related to the primary ovarian tumour as opposed to representing independent primary peritoneal lesions. Nevertheless, the number of invasive implants evaluated by molecular methods in the entire literature is limited.

Implants may also be encountered in the setting of seromucinous borderline tumours, and the same issues for serous tumours pertain. In general, implants do not occur in the setting of borderline mucinous, endometrioid, clear cell or Brenner tumours. In the presence of an 'implant' in association with an ovarian mucinous borderline tumour, an undiagnosed or unsampled primary ovarian mucinous carcinoma or a metastasis from a nongynaecological primary tumour involving the ovary should be excluded.

\section{Serous tubal intraepithelial carcinoma}

Recently, STIC has been implicated in the pathogenesis of extra-uterine HGSC. The evidence indicating that STIC is a precursor of most HGSCs that were formerly considered to be of tubal, ovarian or primary peritoneal origin, as well as guidelines for assigning primary site in cases of advanced stage non-uterine, HGSC, have already been provided (see section above on 'Histological sites of tumour involvement'). STIC comprises a population of cytologically malignant epithelial cells replacing the normal tubal mucosa, most commonly involving the fimbriae, and characterised by increased nuclear to cytoplasmic ratio with rounded nuclei, loss of cell polarity, coarsely clumped chromatin, prominent nucleoli and absence of ciliated cells. Additional features that may be present include epithelial stratification, small fracture lines in the epithelium and tufting and exfoliation from the tubal surface of small epithelial cell clusters.

The diagnostic criteria for STIC have evolved and guidelines for diagnosis, which include the use of p53 and Ki-67 (MIB1) immunostaining, have been published. ${ }^{64-66}$ Use of these criteria results in a high degree of inter-observer diagnostic agreement. In discrete fallopian tube mucosal lesions (usually, but not always, located in the fimbriae) with high-grade atypia in non-ciliated epithelium, the presence of abnormal p53 immunostaining (strong diffuse staining or complete absence of staining) and high Ki-67 labelling index $(\geq 10 \%)$ support a diagnosis of STIC. Although immunostains are a valuable adjunct in the diagnosis of isolated lesions of the fallopian tube, they are usually not needed to diagnosis STIC in the context of advanced-stage HGSC, where comparison between the tubal mucosal lesion and HGSC elsewhere reveals identical cytological features, with high-grade atypia and numerous mitotic figures. Fallopian tube epithelial lesions with atypia that do not meet all the criteria for STIC (e.g., tubal intraepithelial lesion in transition/serous tubal intraepithelial lesion, synonymous terms for such lesions that have some but not all features of STIC) are of uncertain significance at present and these diagnoses should not be used in routine practice; additional research is required to determine the clinical significance, if any, of such lesions. Similarly, p53 signatures should not be reported.

A last consideration is that fallopian tube mucosal involvement by uterine or non-gynaecological primary tumours can occur and mimic STIC. ${ }^{67-69}$ Most cases with unilateral or bilateral HGSC in the ovary and/or STIC or HGSC in the tube but with an endometrial serous intraepithelial or invasive carcinoma will represent adnexal metastases from an endometrial serous carcinoma, and WT1 may be of value in these cases (see section below on 'Immunohistochemical markers'). ${ }^{70} \mathrm{~A}$ diagnosis of STIC always requires consideration of clinical and pathological findings and the exclusion of secondary involvement of the fallopian tube.

\section{Peritoneal cytology}

The results of peritoneal cytology (peritoneal washings or ascitic fluid) are important for the substaging of stage I ovarian tumours (borderline and malignant). Positive peritoneal washings in a stage I tumour signify stage IC3 in the 2014 FIGO staging system. In the previous FIGO staging system, the results of peritoneal cytology were used for the substaging of stage II neoplasms but this is no longer the case. Positive peritoneal cytology in a stage I carcinoma may indicate the need for adjuvant therapy in certain cases.

\section{Lymph nodes}

In the revised 2014 FIGO staging system, metastases involving retroperitoneal lymph nodes, in the absence of peritoneal spread above the pelvic brim or distant metastases, represent stage IIIA1 disease. This stage is further subdivided into stages IIIA1(i) and IIIA1(ii) for nodal metastases $\leq 10 \mathrm{~mm}$ and $>10 \mathrm{~mm}$, respectively. ${ }^{5}$ Formerly, regional node metastases were a criterion for stage IIIC disease and this amendment is 
based upon evidence that patients with only nodal metastases (in the absence of peritoneal disease) have a relatively favourable outcome although it should be noted that the data are based mainly on cases of serous carcinoma. ${ }^{71,72}$ Positive extra-abdominal lymph nodes, including inguinal metastases, represent stage IVB disease.

FIGO specifically restricts the definition of stage IIIA1 disease to retroperitoneal lymph nodes (pelvic and para-aortic) but does not indicate how tumour spread to intraperitoneal nodes (such as those in the mesentery or omentum) should be interpreted, although it would be very unusual to have isolated nodal metastases at these sites. According to FIGO (personal communication), this should be regarded as intra-abdominal disease, i.e., stage IIIC. At present, there are also limited data to justify the subdivision of stage IIIA1 according to the size of the nodal metastases. ${ }^{5}$ It is also not clear how the extent of nodal involvement $(\leq 10 \mathrm{~mm}$ or $>10 \mathrm{~mm})$ should be measured if the diagnosis is based only upon cytological sampling. According to FIGO (personal communication), this should be regarded as stage IIIA(i) disease.

Data on lymph node involvement in borderline ovarian tumours is largely restricted to tumours of serous subtype (SBT) where approximately $25 \%$ of fully staged cases will show positive nodes. ${ }^{73,74}$ Although this finding does not appear to influence overall survival, cases with nodular epithelial tumour aggregates $>1 \mathrm{~mm}$ in extent may show decreased diseasefree survival. ${ }^{75}$ Rarely, low-grade serous carcinoma appears to develop within the lymph nodes of patients with SBT, possibly from foci of endosalpingiosis. ${ }^{76}$

\section{Provisional pathological staging pre-MDTM}

Tumour stage is among the strongest prognostic factors in ovarian carcinoma, ${ }^{77}$ and patients with localised, regional and distant disease have 5-year relative survival rates of 92,72 and $27 \%$, respectively, based on 2014 figures from the United States. ${ }^{78}$

All ovarian carcinomas and borderline tumours and carcinomas of the fallopian tube and peritoneum should be staged using the FIGO 2014 system (Table 5). ${ }^{5}$ The provisional stage, taking into account all the findings in the submitted specimen(s), must be documented in the pathology report but it is recognised that the final FIGO stage should be assigned at the multidisciplinary team/tumour board meeting when the results of all investigations, including radiological, are available. TNM and AJCC staging are optional. At the time of writing this manuscript, neither TNM nor AJCC staging has been updated to take account of the revised FIGO system.

\section{Recommended Data Elements \\ Genetic status}

Table 6 lists the recommended data elements, it is estimated that approximately $10 \%$ of primary tuboovarian and peritoneal carcinomas have a genetic basis ${ }^{79}$ and recent data suggest that this figure may be as high as $17 \%$ for HGSCs specifically. ${ }^{80}$ Germline mutations in BRCA1 and BRCA2 account for the majority of genetically related cases while up to $10 \%$ of such cases are related to LS.

It is acknowledged that definitive genetic status is often not known or information about genetic status is not provided to the pathologist at the time of surgery. Moreover, this information is not essential for the histological assessment and routine reporting of these tumours. Nevertheless, it is recommended that available information on genetic status be recorded for the following reasons:

(1) HGSCs associated with BRCA mutations (germline or somatic) more commonly show certain morphological features, such as solid, endometrioid or transitional-like ('SET') architectural patterns, very marked nuclear atypia and tumour-infiltrating lymphocytes. ${ }^{79,81,82}$ Thus pathologists may be able to correlate the histological findings with any genetic data provided or raise the possibility of $B R C A$ mutation in certain cases with implications regarding improved prognosis, better chemotherapy response and consideration of specific therapeutic regimes such as those including PARP inhibitors. ${ }^{79,80,83}$ Patients with suspected germline BRCA mutations and their relatives, may also be referred for genetic testing and counselling with regard to appropriate screening for BRCA-related neoplasia.

(2) Knowledge of proven or potential hereditary gynaecological cancer predisposition will affect pathological sampling of macroscopically normal tissues. This is most evident in the setting of prophylactic 'risk reduction surgery', especially in patients with known BRCA1 or BRCA2 mutation, where complete examination of tubal and ovarian tissues is mandatory. ${ }^{79}$ The identification of small, macroscopically occult tubal carcinomas and their in situ precursor STIC is much more likely in this setting.

Approximately $2 \%$ of all ovarian cancers are associated with LS due to a germline mutation in one of the genes encoding the DNA mismatch repair (MMR) proteins. In approximately $60 \%$ of women with LS, a gynaecological tumour (endometrial or ovarian) will represent the sentinel cancer. ${ }^{84}$ Endometrioid and clear cell carcinomas occur more frequently in LS and therefore immunohistochemical analysis of MMR proteins or molecular testing for microsatellite instability may be considered in these tumour subtypes or if there is relevant personal or family history of additional LS-related neoplasia. Similar studies may be considered in those patients with synchronous primary ovarian and endometrial endometrioid carcinomas although most such cases are not associated with LS. ${ }^{85}$ It has been suggested that, in a women with an endometrial carcinoma, the presence of a synchronous ovarian clear cell carcinoma may be an indicator of LS. ${ }^{86}$ 
Table 5 FIGO staging of primary ovarian, fallopian tube and peritoneal cancer

\begin{tabular}{|c|c|}
\hline$I$ & umour is confined to ovaries or fallopian tube(s) \\
\hline IA & $\begin{array}{l}\text { Tumour limited to one ovary (capsule intact) or fallopian tube; no tumor on ovarian or fallopian tube surface; no malignant } \\
\text { cells in the ascites or peritoneal washings }\end{array}$ \\
\hline IB & $\begin{array}{l}\text { Tumour limited to both ovaries (capsules intact) or fallopian tubes; no tumour on ovarian or fallopian tube surface; no } \\
\text { malignant cells in the ascites or peritoneal washings }\end{array}$ \\
\hline IC & $\begin{array}{l}\text { Tumour limited to one or both ovaries or fallopian tubes, with any of the following: } \\
\text { IC1 Surgical spill } \\
\text { IC2 Capsule ruptured before surgery or tumour on ovarian or fallopian tube surface } \\
\text { IC3 Malignant cells in the ascites or peritoneal washings }\end{array}$ \\
\hline II & $\begin{array}{l}\text { Tumour involves one or both ovaries or fallopian tubes with pelvic extension (below pelvic brim) or primary peritoneal } \\
\text { cancer }\end{array}$ \\
\hline IIA & Extension and/or implants on uterus and/or fallopian tubes and/or ovaries \\
\hline IIB & Extension to other pelvic intraperitoneal tissues \\
\hline III & $\begin{array}{l}\text { Tumour involves one or both ovaries or fallopian tubes, or primary peritoneal cancer, with cytologically or histologically } \\
\text { confirmed spread to the peritoneum outside the pelvis and/or metastasis to the retroperitoneal lymph nodes }\end{array}$ \\
\hline IIIA1 & Positive retroperitoneal lymph nodes only (cytologically or histologically proven): \\
\hline IIIA1(i) & Metastasis up to $10 \mathrm{~mm}$ in the greatest dimension \\
\hline IIIA1(ii) & Metastasis $>10 \mathrm{~mm}$ in the greatest dimension \\
\hline IIIA2 & $\begin{array}{l}\text { Microscopic extrapelvic (above the pelvic brim) peritoneal involvement with or without positive retroperitoneal lymph } \\
\text { nodes }\end{array}$ \\
\hline IIIB & $\begin{array}{l}\text { Macroscopic peritoneal metastasis beyond the pelvis up to } 2 \mathrm{~cm} \text { in the greatest dimension, with or without metastasis to the } \\
\text { retroperitoneal lymph nodes }\end{array}$ \\
\hline IIIC & $\begin{array}{l}\text { Macroscopic peritoneal metastasis beyond the pelvis }>2 \mathrm{~cm} \text { in the greatest dimension, with or without metastasis to the } \\
\text { retroperitoneal lymph nodes (includes extension of tumor to capsule of liver and spleen without parenchymal involvement } \\
\text { of either organ) }\end{array}$ \\
\hline IV & Distant metastasis excluding peritoneal metastases \\
\hline IVA & Pleural effusion with positive cytology \\
\hline IVB & $\begin{array}{l}\text { Parenchymal metastases and metastases to extra-abdominal organs (including inguinal lymph nodes and lymph nodes } \\
\text { outside of the abdominal cavity) }\end{array}$ \\
\hline
\end{tabular}

Site of primary tumour:

Primary tumour, ovary (OV).

Primary tumour, fallopian tube (FT).

Primary tumour, peritoneum (P).

Undesignated: site of primary tumour cannot be assessed (X).

Table 6 Recommended data items for pathological reporting of ovarian, fallopian tube and primary peritoneal carcinomas

\begin{tabular}{llll}
\hline Clinical & Macroscopic & Microscopic & Other \\
\hline Genetic status & Tumour dimensions & Pattern of invasion (for mucinous carcinomas only) & $\begin{array}{c}\text { Ancillary studies: } \\
\text { Immunohistochemical markers } \\
\text { Molecular data }\end{array}$ \\
& & Carcinosarcoma subtypes & Tumour grade (for mucinous carcinomas) \\
& & Borderline tumour: \\
& Special features & Intraepithelial carcinoma for mucinous borderline \\
& tumour & \\
& Response to neoadjuvant therapy \\
& Coexistent pathology & \\
&
\end{tabular}

\section{Tumour dimensions}

There is little or no published evidence to suggest that size of the primary tumour is of prognostic significance, and size is not important for staging or management. The principal reason for recording the tumour dimensions, especially the maximum diameter, is to provide evidence that the tumour has been adequately sampled for histology. There are no evidence-based guidelines as to the optimal sampling of solid or cystic ovarian tumours. By convention, however, most pathologists sample one block per $\mathrm{cm}$ of maximum tumour diameter in solid tumours. It has been recommended that soft tissue tumours $<2 \mathrm{~cm}$ in diameter be blocked in their entirety and that a minimum of 1 section per $\mathrm{cm}$ of maximum diameter be examined for larger tumours. ${ }^{87}$ These same recommendations appear in cancer data sets for tumours at a range of anatomical sites.

Adequate sampling of ovarian tumours is important for a number of reasons; for example, to identify 
small foci of carcinosarcoma in ovarian carcinomas, histological heterogeneity (e.g., different epithelial subtypes in mixed carcinomas) and to identify foci of microinvasion or invasion in borderline tumours. Adequate sampling may also assist in identifying diagnostic areas in poorly differentiated neoplasms or features, which suggest a particular tumour subtype. For example, the presence of squamous differentiation may help to confirm an endometrioid neoplasm, and identification of endometriosis supports a diagnosis of endometrioid, clear cell or seromucinous tumours.

It is recognised that ovarian mucinous neoplasms may exhibit considerable intratumoral heterogeneity with an admixture of benign, borderline and malignant areas. One study, which assessed the 'adequacy' of sampling of one section per 1-2 $\mathrm{cm}$ of maximum tumour diameter in epithelial ovarian neoplasms, ${ }^{88}$ confirmed mucinous carcinomas to display more histological variation than serous carcinomas. The authors concluded that more extensive sampling was required in borderline tumours to exclude foci of invasion. According to the recommendations of the 2004 Bethesda Workshop for borderline ovarian tumours, ${ }^{89}$ all borderline tumours should be well sampled-at least 1 block per centimetre of maximum tumour diameter for neoplasms $<10 \mathrm{~cm}$ and 2 sections per centimetre for larger tumours (excluding smooth-walled cystic foci). The recommendation that there should be more extensive sampling of larger tumours, especially those of mucinous type, reflects their greater likelihood of harbouring foci of invasive carcinoma. Additional sampling of mucinous borderline tumours is also recommended when histological features such as intraepithelial carcinoma or microinvasion are identified in the original sections. Similarly, additional sampling in serous borderline tumours is recommended when micropapillary areas or microinvasion are present in initial sections as such neoplasms are more likely to harbour invasive foci.

Seidman et $a 9^{90}$ suggested that, in mucinous ovarian tumours, tumour size may be helpful in determining whether the ovarian neoplasm is primary or metastatic. The authors found that unilateral mucinous carcinomas $\geq 10 \mathrm{~cm}$ in diameter were more likely be primary than metastatic. Similar findings were reported by others. ${ }^{91}$

\section{Block identification key}

The origin/designation of all tissue blocks should be recorded and it is preferable to document this information in the final pathology report. This is particularly important should the need for internal or external review arise. The reviewer needs to be clear about the origin of each block in order to provide an informed specialist opinion. If this information is not included in the final pathology report, it should be available on the laboratory computer system and relayed to the reviewing pathologist.
Recording the origin/designation of tissue blocks also facilitates retrieval of blocks, for example, for further immunohistochemical or molecular analysis, research studies or clinical trials.

\section{Pattern of invasion}

It is controversial as to whether the pattern of invasion in stage 1 mucinous ovarian carcinoma has prognostic significance. ${ }^{92-97}$ The expansile/confluent/non-destructive pattern of invasion is characterised by architecturally complex glands, cysts or papillae lined by atypical epithelium with minimal to no intervening stroma. The destructive/infiltrative pattern is characterised by haphazardly arranged glands, tubules, nests and cords of malignant cells infiltrating stroma with an associated oedematous, inflammatory or desmoplastic response. Although several studies have shown the expansile pattern to herald a better prognosis, a recent population-based registry study of mucinous ovarian carcinomas was not able to prognosticate based on the distinction between the two patterns of invasion. ${ }^{92-97}$ It is recommended that the pattern of invasion in mucinous ovarian carcinomas be recorded.

\section{Carcinosarcoma subtypes}

There is little published evidence suggesting any prognostic significance of the different morphological subtypes within ovarian carcinosarcomas (evidence exists for uterine carcinosarcomas). ${ }^{98-100}$ However, in view of the paucity of studies, the ICCR recommends that it would be useful to record the percentage of the epithelial and mesenchymal elements as well as the subtypes of the epithelial and mesenchymal components. However, the panel recognises that on occasions it may be difficult or even impossible to classify the epithelial and mesenchymal subtypes given that overlapping and mixed patterns not infrequently occur in this tumour type. This is a recommended rather than a required element and collection of these data may be informative for the future regarding the prognosis and management of these neoplasms. ${ }^{98-100}$

\section{Tumour grade}

Mucinous carcinoma. There is also little evidence for grading mucinous carcinomas, although oncologists often ask for a tumour grade. The ICCR panel suggests that if grading of these neoplasms is undertaken (a recommended rather than required element in the case of mucinous carcinomas), the same grading system for endometrioid carcinomas should be used. Malignant mural nodules in ovarian mucinous neoplasms are automatically grade 3 .

\section{Borderline tumours}

Intraepithelial carcinoma. In mucinous borderline tumours, intraepithelial carcinoma is diagnosed in non-invasive foci with marked nuclear atypia. ${ }^{29,57,58}$ However, the reproducibility of this diagnosis has not been formally analysed. 
Table 7 Chemotherapy Response Score (CRS)

\begin{tabular}{lll}
\hline Score & Criterion & Tumour regression grading \\
\hline 1 & $\begin{array}{l}\text { Mainly viable tumour with minimal regression-associated fibro-inflammatory } \\
\text { changes limited to a few foci } \\
\text { Multifocal or diffuse regression-associated fibro-inflammatory changes, with viable } \\
\text { tumour ranging from diffuse sheets, streaks or nodules, to extensive regression with } \\
\text { multifocal but easily identifiable residual tumour }\end{array}$ & Portial tumour response \\
& $\begin{array}{l}\text { Mainly regression, with few irregularly scattered individual tumour cells or cell } \\
\text { groups (all measuring }<2 \mathrm{~mm}), \text { or no residual tumour identified }\end{array}$ & $\begin{array}{l}\text { Complete or near-complete } \\
\text { response }\end{array}$
\end{tabular}

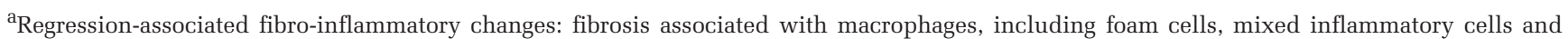
psammoma bodies, to be distinguished from tumour-related inflammation or desmoplasia.

Response to neoadjuvant therapy

There is no recommended or agreed system for tumour regression grading (TRG) of ovarian/tubal/ peritoneal carcinomas that have been treated with neoadjuvant chemotherapy (this largely applies to pelvic HGSCs) despite the fact that oncologists often request this information because it is potentially a helpful morphological marker to assess the response to neoadjuvant treatment after surgery and identify patients who may be eligible for entry into trials. TRG has been shown to provide valuable prognostic information in patients with carcinomas of the breast, stomach, oesophagus and colorectum who have been treated with neoadjuvant chemotherapy and serves as a morphological marker to guide further treatment after surgery. ${ }^{101-105}$ The applicability of several well-known and widely used systems for TRG has been considered for pelvic gynaecological carcinomas. Some of the systems that are used for breast carcinoma are unduly complex and include the separate assessment of both the primary tumour and involved lymph nodes. ${ }^{106-108}$ Most of the different TRG systems for gastrointestinal tumours are relatively simple to use, ${ }^{102,109,110}$ although the reported reproducibility of these systems is variable. ${ }^{111-114}$ TRG is usually applied to the primary site of unifocal tumours in the breast and gastrointestinal tract. In contrast, pelvic HGSCs tend to affect multiple intra-abdominal sites in addition to the primary site of origin. They also typically evoke a desmoplastic host reaction and the inclusions of fibrosis as a criterion for tumour regression has the potential to provide misleading data.

Four studies have assessed tumour regression after neoadjuvant chemotherapy in advanced-stage ovarian cancer and all showed a correlation between response and survival; however, all used different scoring criteria, did not validate their criteria in an independent series of cases and did not assess reproducibility of their criteria. ${ }^{115-118}$ A more recent study has tested and validated the prognostic significance of response criteria and assessed reproducibility in two independent series of high-grade pelvic serous carcinoma. ${ }^{118,119}$ The latter study suggests that a three-tier scoring system (the Chemotherapy Response Score (CRS)) is most reproducible and that the system is simple and easy for all pathologists to apply, irrespective of their level of experience in gynaecological pathology. In this study, the prognostic significance of the CRS as applied to omental tumour deposits was superior to the CRS of the primary tumour. The study (which included 60 patients in the test cohort and 71 in the validation cohort) used a modification of the Dworak system ${ }^{110}$ and demonstrated good inter-observer reproducibility and significant association with clinical outcome. Although further studies are needed to confirm the findings, this is the grading system currently recommended by the ICCR. The method is as follows:

(1) Scoring should be carried out on a single H\&Estained section (refer to discussion of omental sampling in section on 'Macroscopic description of omentum').

(2) A single block of involved omental tissue that shows the least response to chemotherapy should be selected (if there is no residual omental tumour a CRS score of 3 is given-Table 7)

(3) The amount of viable tumour should be assessed; this may or may not show degenerative changes in the form of nuclear atypia, smudging of the nuclear chromatin and cytoplasmic clearing.

(4) A three-tier system for CRS should be used (Table 7).

(5) The presence of fibrosis may be helpful in marking the site of previous tumour infiltration.

(a) When found in the absence of tumour, fibrosis is likely to indicate regression.

(b) If fibrosis occurs in association with tumour, this may simply reflect tumour-associated desmoplasia rather than regression.

(c) However, when fibrosis in association with tumour is accompanied by an inflammatory response (so-called 'fibro-inflammatory' response-fibrosis with associated macrophages and a mixed population of inflammatory cells), this indicates regression.

(d) Psammoma bodies may mark the site of previous tumour and can sometimes appear more numerous because their density increases in areas where tumour has disappeared. 
(6) As a guide, $>95 \%$ of tumour should be viable for a score of 1 , and $<5 \%$ for a score of 3 .

(7) In studies to date, using this system or a closely related system, a difference in prognosis was shown only when tumours with a CRS score of 1 or 2 were compared with those having a CRS score of $3 .{ }^{118,119}$ However, the ICCR recommends use of the three-tier system to gather more data for future studies.

(8) Note that this system has only been applied to HGSCs to date.

\section{Coexistent pathology}

Borderline and malignant endometrioid, clear cell and seromucinous ovarian tumours may arise from endometriosis. Thus the presence of endometriosis, although not of prognostic or therapeutic significance, particularly if contiguous with the tumour, may assist in determining the histotype in problematic cases. The presence of endometriosis may also support a primary ovarian origin rather than metastasis from a primary uterine carcinoma of the same cell type.

\section{Immunohistochemical markers}

Immunohistochemistry has many important applications in the field of ovarian neoplasia. ${ }^{120-122}$ There are a number of scenarios where immunohistochemical markers may assist in establishing a diagnosis of a primary ovarian epithelial malignancy or in tumour subtyping. It is beyond the scope of this data set to present a detailed analysis of every scenario but major uses of immunohistochemistry are discussed. In general, panels of markers are better than reliance on individual markers and it should be remembered that no marker is totally specific or sensitive for any tumour type. Unexpected positive and negative staining reactions may occur and the results of immunohistochemical studies should always be interpreted in conjunction with the clinical, gross and microscopic features.

Markers of use in typing ovarian carcinomas. Although most primary ovarian carcinomas are straightforward to type, on occasion it is difficult to distinguish between a HGSC and a high-grade endometrioid carcinoma or between a clear cell carcinoma and clear cell areas within a HGSC or an endometrioid carcinoma. A panel of markers may help that should be tailored depending on the differential diagnosis. Approximately $80-90 \%$ of serous carcinomas (low grade and high grade) are positive with WT1, usually with diffuse immunoreactivity. ${ }^{70,123-127}$ In contrast, endometrioid and clear cell carcinomas are usually negative, although a small percentage of endometrioid carcinomas are positive. ${ }^{128}$ HGSCs exhibit aberrant 'mutation-type' staining with p53 (see below) while low-grade serous carcinomas, clear cell carcinomas and most endometrioid carcinomas exhibit 'wild-type' staining (focal and heterogenous); some high-grade endometrioid carcinomas exhibit aberrant p53 staining. p16 is diffusely positive ('block-type' staining) in most HGSCs while most low-grade serous carcinomas, clear cell carcinomas and endometrioid carcinomas exhibit patchy immunoreactivity. ${ }^{129}$ Clear cell carcinomas usually exhibit diffuse strong nuclear staining with hepatocyte nuclear factor 1-beta while other primary ovarian epithelial neoplasms are usually negative or focally positive. ${ }^{130}$ Napsin $\mathrm{A}$ is also a useful marker of clear cell carcinomas. ${ }^{131}$ ER is positive in most high-grade and low-grade serous carcinomas and endometrioid carcinomas while clear cell carcinomas are usually negative. Some of these markers have helped establish that most neoplasms, which were previously classified as mixed high-grade serous and endometrioid and mixed high-grade serous and clear cell, represent HGSCs with pseudoendometrioid areas and areas of cytoplasmic clearing.

On occasion, especially in a biopsy specimen, it may be problematic to differentiate between a lowgrade and a high-grade serous small carcinoma. The most useful marker in this scenario is p53 ('mutation-type' staining in HGSC; 'wild-type' staining in low-grade serous carcinoma).

Distinction between primary and secondary/metastatic ovarian adenocarcinoma. The distinction between a primary ovarian adenocarcinoma and metastatic adenocarcinoma from various sites may be problematic. ${ }^{132}$ Metastatic colorectal adenocarcinomas may mimic an endometrioid carcinoma or a mucinous neoplasm of intestinal type, either borderline or malignant. In the distinction between an ovarian endometrioid adenocarcinoma and a metastatic colorectal adenocarcinoma with a pseudoendometrioid pattern, a panel of markers may assist. Although there may be immunophenotypic overlap of individual markers, primary ovarian endometrioid carcinomas are usually positive with CK7, ER, CA125 and PAX8 and negative with CK20, CEA and CDX2 while the converse immunophenotype is the rule in metastatic colorectal adenocarcinomas. ${ }^{120-122}$ In distinguishing between a primary ovarian mucinous tumour and a metastatic colorectal adenocarcinoma, immunohistochemistry is less helpful. This is because many primary ovarian mucinous neoplasms exhibit CK20 positivity, usually focal but sometimes widespread. They are also commonly positive, sometimes diffusely so, with CEA, CDX2 and CA19.9. The expression of these enteric markers is a reflection of intestinal differentiation in primary ovarian mucinous neoplasms. However, the pattern of coordinate expression of CK7/CK20 may assist in distinguishing between a primary ovarian mucinous tumour and a metastatic colorectal adenocarcinoma with a mucinous appearance. Although either marker can be positive in both tumours, primary ovarian mucinous neoplasms are often diffusely positive with CK7 while CK20 is variable; conversely metastatic colonic adenocarcinoma is usually diffusely positive with CK20 and focally positive with CK7 when this marker is 
expressed. Thus CK7 immunopositivity is typically of greater extent than CK20 immunopositivity in primary ovarian mucinous tumours and CK20 staining is more extensive than CK7 in metastatic colonic adenocarcinoma. ${ }^{133}$

Metastatic pancreatic or biliary adenocarcinoma may mimic a primary ovarian mucinous neoplasm of intestinal type, either borderline or malignant, and immunohistochemistry is of limited value. Most commonly, these tumour types are diffusely positive with CK7 while CK20 is variable, being negative, focally or diffusely positive. CEA, CA19.9 and CDX2 may be positive. An absence of staining with DPC4 (DPC = deleted in pancreatic cancer) may be a useful pointer towards a pancreatic adenocarcinoma as this nuclear transcription factor is inactivated in about $50 \%$ of pancreatic adenocarcinomas with the result that approximately half of these are negative. ${ }^{134}$ Conversely, DPC4 is expressed in virtually all primary ovarian mucinous neoplasms.

Metastatic breast carcinomas of ductal type may mimic a HGSC or an endometrioid carcinoma. It is not an uncommon scenario that a patient with a history of breast carcinoma is found to have a pelvic mass or a disseminated peritoneal malignancy. In most cases, this will represent a new tubo-ovarian HGSC; such patients may or may not have underlying $B R C A 1 / 2$ mutation. In distinguishing between a metastatic breast carcinoma and a tubo-ovarian HGSC, markers which may be useful are PAX8, CA125 and WT1 (usually positive in HGSCs and negative in breast carcinomas, although occasionally the latter are CA125 or WT1 positive) and GCDFP15, mammoglobin and GATA3 (usually negative in HGSCs and positive in breast carcinomas). ${ }^{135-137} \mathrm{~A}$ similar panel of markers is useful in the distinction between an endometrioid carcinoma and a metastatic breast carcinoma, although WT1 is negative in endometrioid carcinomas and a proportion of these may be mammoglobin positive. ${ }^{138}$

Rarely, a metastatic cervical adenocarcinoma of usual type (HPV related) in the ovary may mimic a primary ovarian mucinous or endometrioid neoplasm. ${ }^{139}$ Diffuse p16 immunoreactivity in such cases may be useful in suggesting a metastatic cervical adenocarcinoma.

Distinction between ovarian endometrioid carcinoma and sex cord-stromal tumour. Some primary ovarian carcinomas, especially of endometrioid type, may closely mimic an ovarian sex cord-stromal tumour, either a granulosa cell tumour or a Sertoli cell tumour. Conversely, some Sertoli-Leydig cell tumours have a pseudoendometrioid appearance and can mimic an endometrioid neoplasm. ${ }^{140}$ Markers that are useful to distinguish between an endometrioid neoplasm and a sex cord-stromal tumour include inhibin, calretinin and steroidogenic factor-1 (SF-1; positive in sex cord-stromal tumours) and epithelial membrane antigen and CK7 (positive in epithelial neoplasms). ${ }^{120-122,140-142}$
Diagnosis of STIC. Biomarkers are not necessary if the features are unequivocally those of STIC but if there is diagnostic uncertainty, both p53 and MIB1 staining should be performed. ${ }^{143}$ The cells must exhibit aberrant p53 staining (see definition below). The MIB1 proliferative index is increased, typically in the region of $40 \%$ to nearly $100 \%$, with most cases showing focal areas $>70 \%$. However, some cases of STIC exhibit a lower MIB1 proliferation index and it has been suggested that at least $10 \%$ of the nuclei should be positive for a diagnosis of STIC in cases where immunohistochemistry is undertaken (morphological features and aberrant p53 staining are also needed). ${ }^{143}$

Two patterns of aberrant p53 staining. There is significant variability among pathologists in the interpretation of p53 staining. Pathologists are often unaware that many normal tissues and tumours unassociated with TP53 abnormalities express p53 protein. Such staining is usually focal and weak and somewhat variable from area to area (referred to as 'wild-type' p53 staining), although on occasions many of the nuclei are positive, albeit with variable intensity. The degree of positive staining can be affected by varying the antibody concentration used. ${ }^{144}$ This pattern of staining is found in many normal tissues (non-neoplastic epithelia, stromal and lymphoid cells, which can act as an internal positive control) and neoplasms not related to TP53 mutation. Rather than this 'wild-type' staining, it is the diffuse intense pattern of nuclear immunoreactivity that should be interpreted as 'positive' and that is correlated with TP53 missense mutations. Typically, in excess of $75 \%$ and sometimes almost all of the nuclei are intensely positive. It should also be appreciated that totally absent p53 staining (as stated, there is usually an in-built positive control with 'wild-type' staining of non-neoplastic tissues) is also indicative of aberrant p53 immunoreactivity. ${ }^{145,146}$ This pattern of immunoreactivity is in keeping with a null (including non-sense, frame shift or splice site) TP53 mutation resulting in complete absence of detectable protein. To summarise, it is not simply negative or positive staining but rather patterns of p53 immunoreactivity that are of importance. Diffuse intense nuclear immunoreactivity and totally absent staining ('all or nothing') are aberrant patterns ('mutation-type' staining) and in keeping with an underlying TP53 mutation, whereas 'wild-type' staining is not.

Distinction between ovarian and uterine carcinoma. A not uncommon scenario is simultaneous involvement of the uterine corpus and one or both ovaries by an adenocarcinoma. Most commonly, the adenocarcinomas are endometrioid in type but sometimes they are serous. ${ }^{147,148}$ With endometrioid adenocarcinomas involving the uterus and one or both ovaries, immunohistochemistry is of little or no value in ascertaining the relationship between the tumours as the immunophenotype of a primary 
ovarian and uterine endometrioid adenocarcinoma is essentially identical.

With a serous carcinoma involving the uterus and one or both ovaries, WT1 staining may be of some value in distinguishing between a uterine serous carcinoma with metastasis to the ovary, metastasis from the ovary/ tube to the endometrium ('drop metastasis') and independent synchronous neoplasms, the latter being unlikely. ${ }^{70,123-127,149}$ Most tubo-ovarian serous carcinomas exhibit diffuse nuclear positivity with WT1, whereas most uterine serous carcinomas are negative. However, there is some overlap in that a proportion of uterine serous carcinomas are WT1 positive (the percentage has varied between studies) and a small percentage of tubo-ovarian HGSCs are WT1 negative. ${ }^{70,123-127}$ It can be summarised that, although there is some overlap, diffuse WT1 positivity in a serous neoplasm favours a tubo-ovarian origin. In contrast, negative staining is a pointer towards a primary uterine neoplasm.

Distinction between serous and mesothelial proliferation. On occasion, it may be difficult to distinguish between a serous proliferation (borderline or malignant) and a mesothelial proliferation (reactive or neoplastic). Florid reactive mesothelial proliferation may occur in association with endometriosis and mimic an endometrioid carcinoma. ${ }^{150} \mathrm{~A}$ suggested panel of markers in this situation would include BerEP4, ER and PAX8 (usually positive in serous proliferations and endometrioid carcinomas) and calretinin and CK5/6 (usually positive in mesothelial proliferations). WT1 is usually positive in both serous and mesothelial proliferations.

\section{Molecular data}

Ovarian carcinomas represent a heterogeneous group of tumours. ${ }^{151-156}$ In recent years, molecular pathology has been instrumental in demonstrating that ovarian carcinomas are not a single entity but a group of tumours with diverse morphology, natural history and pathogenesis. ${ }^{157}$ Although molecular investigations at present do not have a significant role in diagnosis, prediction of prognosis or determination of treatment in ovarian, tubal and peritoneal carcinomas, this may change in the future.

HGSCs are chromosomally unstable tumours, in which TP53 mutations are ubiquitous. Germ-line or sporadic, genetic or epigenetic, alterations in BRCA1 and BRCA2 also occur. A pathogenetic model has been proposed, starting with early TP53 alteration, followed by BRCA1 loss, leading to deficiency in homologous recombination repair of double-strand breaks, triggering chromosomal instability with gene copy number variation. The Cancer Genome Atlas performed an integrated genomic analysis of 489 high-grade ovarian serous carcinomas. ${ }^{153}$ Mutations in TP53 were seen in 96\% of the cases. There was a low prevalence, but there were statistically recurrent somatic mutations in nine further genes, including $N F 1, B R C A 1, B R C A 2$,
$R B 1$ and CDK12. Copy number alterations and promoter hypermethylation events were detected in 168 genes. The most common amplifications were detected in CCNE1, MYC and MECOM. Deletions were identified in RB1, NF1 and PTEN. Hierarchical clustering analysis identified four transcriptional subtypes, three microRNA subtypes, four promoter methylation subtypes and a transcriptional signature associated with survival. In all, $33 \%$ of the tumours showed alterations in BRCA genes, either somatic or germline mutations or promoter hypermethylation.

Low-grade serous carcinomas are closely related to serous borderline tumours and show frequent mutations in KRAS and BRAF, which are mutually exclusive events.

The molecular events in endometrioid adenocarcinoma are similar to the uterine counterpart. The main molecular alterations are: microsatellite instability $(12-20 \%)$, and mutations in the PTEN (20\%), KRAS and PIK3CA genes. Mutations in exon 3 of CTNNB1 with nuclear accumulation of betacatenin occur in $38-50 \%$ of cases. Mutation of the ARID1A gene has recently been described. ${ }^{155}$

Clear cell carcinoma shows frequent PIK3CA mutations and also PTEN inactivation. Alterations in KRAS and Tp53 are unusual. Mutation of the ARID1A gene and loss of the corresponding protein BAF250a has recently been described, occurring in $50 \%$ of the tumours. ${ }^{155}$ They also show upregulation of HNF-1-beta.

Mucinous carcinomas frequently contain KRAS mutations. ${ }^{156}$ In mucinous tumours with areas of carcinoma admixed with foci of benign or borderline tumour, KRAS mutations have been demonstrated in all components, suggesting that this represents an early event during tumorigenesis. However, in general, KRAS mutations are more frequent in carcinomas in comparison with benign mucinous tumours. Amplification of $c$-erbB2 is present in some mucinous carcinomas.

\section{Existing International Data Set Element Considered but not Included}

\section{Lymphovascular invasion (LVI)}

LVI has traditionally been included in all ovarian cancer data sets. However, the expert panel decided that there was no evidence for this as a required element and it was the view of the panel that it should not be included as a recommended element either. Relatively few studies have assessed the prognostic significance of LVI in ovarian ${ }^{98,158-160}$ or fallopian tube ${ }^{161-164}$ carcinoma. Although some studies have demonstrated a correlation between LVI and nodal metastases, tumour stage and highgrade serous histological type,${ }^{158}$ none has shown a statistically significant association with overall survival upon multivariate analysis. ${ }^{98,158-160}$ A few studies of fallopian tube carcinoma have found LVI to be prognostically important and to correlate with depth of invasion of the tubal wall and lymph node metastases. ${ }^{161,163,164}$ However, other studies 
did not show LVI to be of independent prognostic significance. ${ }^{162,164}$ Given the limited number of studies, the relatively small number of patients with LVI in most studies and the absence of any assessment of the level of interobserver reproducibility of a diagnosis of LVI in any study, the available evidence is insufficient to recommend the reporting of LVI.

\section{Discussion}

This data set was developed for resection specimens of primary borderline and malignant epithelial tumours of the ovary, fallopian tube and peritoneum. Nonepithelial neoplasms and other primary peritoneal tumours, such as mesothelioma, were not included. One of the main challenges for the ICCR panel was to coordinate the development of the data set with the publications of the revised 2014 FIGO staging system for ovary, fallopian tube and peritoneum ${ }^{5}$ and the revised 2014 WHO Classification of Tumours of the Female Reproductive Organs. ${ }^{30}$ This was achieved, ensuring that the data set is 'up to date' with inclusion of information from these important recent publications. In the future, the ICCR has agreed to align the development of cancer data sets with the IARC revision of the 'Blue book' series, and data set development has already commenced for the full range of cardiothoracic tumours, which is the next WHO tumour classification monograph that the IARC will be revising and publishing in 2015. Unfortunately, the revised classification of tumours of the Female Reproductive Organs was published in 2014 by the IARC $^{30}$ prior to the ICCR decision to align data set development with the IARC Blue books. Apart from the existing ICCR cancer data set on endometrial tumours (which is currently being updated to take into account the changes in classification of endometrial tumours), the remaining gynaecological cancer data sets in the current cycle are therefore being produced separately from the 'Blue book' series. The International Society of Gynecological Pathologists has agreed to develop the next cancer data set on cervical tumours in partnership with the ICCR.

In this data set, the ICCR panel dealt with a few controversial issues. The panel recommends grading of different morphological subtypes of carcinoma using different grading systems (see section on 'Tumour grade'). Grading of ovarian carcinomas is controversial with some using universal grading systems and others employing different grading for different tumour types. The panel also introduces two major new recommendations in controversial areas where there is minimal published literature: (i) designating the site of origin of extrauterine HGSC when there is more than one site of tumour involvement and (ii) grading of tumour regression in HGSC following neoadjuvant chemotherapy. Both of these recommendations were being concurrently developed by different groups of pathologists, which included individual members of the ICCR panel. In the case of designating the site of origin of HGSC, the recommendations have been recently published. ${ }^{24}$ In the case of TRG, the panel has endorsed a CRS that is reproducible and easy to use and has been shown to be of prognostic relevance. ${ }^{119}$ The expert panel was also able to exclude an outdated and unvalidated data item (LVI). Although traditionally included in most (or all) ovarian cancer data sets, the panel, following a literature review, decided that there was no evidence for the inclusion of this parameter.

It is important that the elements included in structured pathology reports are recorded using consistent value responses to facilitate comparison of data between different centres and countries. It is also important to note that the data sets are not restrictive and, according to local needs, additional data items may be included in the reporting proforma. All structured pathology reports must also include the facility for free text comments. The ICCR will also ensure that their data sets are reviewed and updated on a regular basis as new literature is published.

\section{Conclusion}

This review describes the process of development of the ICCR data set for reporting of ovary, fallopian tube and primary peritoneal carcinomas. The data set has been developed by an international panel of expert gynaecological pathologists and defines those required elements that must be recorded in pathology reports and recommended elements that may be included. The data set also lists the response values for each element and explanatory notes/commentaries are provided. Such internationally agreed, evidence-based, structured pathology data sets are important to facilitate comparison of data between countries and will be important for cancer research and benchmarking.

\section{Disclosure/conflict of interest}

The authors declare no conflict of interest.

\section{References}

1 Kench J, Delahunt B, Griffiths DF et al. Dataset for reporting of prostate carcinoma in radical prostatectomy specimens: recommendations from the International Collaboration on Cancer Reporting. Histopathology 2013;62:203-218.

2 McCluggage WG, Colgan T, Duggan M et al. Data Set for Reporting of Endometrial Carcinomas: Recommendations From the International Collaboration on Cancer Reporting (ICCR) Between United Kingdom, United States, Canada, and Australasia. Int J Gynecol Pathol 2012;32:45-65.

3 Scolyer RA, Judge MJ, Evans A et al. Data set for pathology reporting of cutaneous invasive melanoma 
recommendations from the International Collaboration on Cancer Reporting (ICCR). Am J Surg Pathol 2013;37:1797-1814.

4 Jones KD, Churg A, Henderson DW et al. Data set for reporting of lung carcinomas: recommendations from International Collaboration on Cancer Reporting. Arch Pathol Lab Med 2013;137:1054-1062.

5 Prat J, FIGO Committee on Gynecologic Oncology. Staging classification for cancer of the ovary, fallopian tube, and peritoneum. Int J Gynaecol Obstet 2014;124: $1-5$.

6 Merlin T, Weston A, Tooher R. Extending an evidence hierarchy to include topics other than treatment: revising the Australian 'levels of evidence'. BMC Med Res Methodol 2009;9:34.

7 Suh DH, Kim JW, Kim K et al. Major clinical research advances in gynecologic cancer in 2012. J Gynecol Oncol 2013;24:66-82.

$8 \mathrm{Kim} \mathrm{HS}$, Ahn JH, Chung HH et al. Impact of intraoperative rupture of the ovarian capsule on prognosis in patients with early-stage epithelial ovarian cancer: a meta-analysis. Eur J Surg Oncol 2013;39:279-289.

9 Vergote I, De Brabanter J, Fyles A et al. Prognostic importance of degree of differentiation and cyst rupture in stage I invasive epithelial ovarian carcinoma. Lancet 2001;357:176-182.

10 Bakkum-Gamez JN, Richardson DL, Seamon LG et al. Influence of intraoperative capsule rupture on outcomes in stage I epithelial ovarian cancer. Obstet Gynecol 2009;113:11-17.

11 Seidman JD, Yemelyanova AV, Khedmati F et al. Prognostic factors for stage I ovarian carcinoma. Int J Gynecol Pathol 2010;29:1-7.

12 Dembo AJ, Davy M, Stenwig AE et al. Prognostic factors in patients with stage I epithelial ovarian cancer. Obstet Gynecol 1990;75:263-273.

13 Ahmed FY, Wiltshaw E, A'Hern RP et al. Natural history and prognosis of untreated stage I epithelial ovarian carcinoma. J Clin Oncol 1996;14:2968-2975.

14 Timmers PJ, Zwinderman AH, Teodorovic I et al. Clear cell carcinoma compared to serous carcinoma in early ovarian cancer: same prognosis in a large randomized trial. Int J Gynecol Cancer 2009;19: 88-93.

15 Higashi M, Kajiyama H, Shibata K et al. Survival impact of capsule rupture in stage I clear cell carcinoma of the ovary in comparison with other histological types. Gynecol Oncol 2011;123:474-478.

16 Gottheil S, McGee J. Endometrioid ovarian carcinoma during pregnancy presenting with acute rupture. J Obstet Gynaecol Can 2013;35:1020-1022.

17 Colgan TJ, Murphy J, Cole DE et al. Occult carcinoma in prophylactic oophorectomy specimens: prevalence and association with BRCA germline mutation status. Am J Surg Pathol 2001;25:1283-1289.

18 Piek JM, van Diest PJ, Zweemer RP et al. Dysplastic changes in prophylactically removed fallopian tubes of women predisposed to developing ovarian cancer. J Pathol 2001;195:451-456.

19 Kindelberger DW, Lee Y, Miron A et al. Intraepithelial carcinoma of the fimbria and pelvic serous carcinoma: evidence for a causal relationship. Am J Surg Pathol 2007;31:161-169.

20 Kuhn E, Meeker A, Wang TL et al. Shortened telomeres in serous tubal intraepithelial carcinoma: an early event in ovarian high-grade serous carcinogenesis. Am J Surg Pathol 2010;34:829-836.
21 Kuhn E, Bahadirli-Talbot A, Kurman R et al. CCNE1 amplification may precede centrosome number abnormality in progression from serous tubal intraepithelial carcinoma to high-grade serous carcinoma. Mod Pathol 2013;26:283A.

22 Garg K, Rabban J. Practical value of systematic and complete examination of fallopian tubes in unselected women undergoing salpingectomy for benign indications: results of a prospective study. Mod Pathol 2013;26:276A.

23 Przybycin CG, Kurman RJ, Ronnett BM et al. Are all pelvic (nonuterine) serous carcinomas of tubal origin? Am J Surg Pathol 2010;34:1407-1416.

24 Singh N, Gilks CB, Wilkinson N et al. Assignment of primary site in high-grade serous tubal, ovarian and peritoneal carcinoma: a proposal. Histopathology 2014;65:149-154.

25 Wethington SL, Park KJ, Soslow RA et al. Clinical outcome of isolated serous tubal intraepithelial carcinomas (STIC). Int J Gynecol Cancer 2013;23: 1603-1611.

26 Bloss JD, Liao S, Buller RE et al. Extraovarian peritoneal serous papillary carcinoma: a case-control retrospective comparison to papillary adenocarcinoma of the ovary. Gynecol Oncol 1993;50:347-351.

27 Doig T, Monaghan H. Sampling the omentum in ovarian neoplasia: when one block is enough. Int J Gynecol Cancer 2006;16:36-40.

28 Usubütün A, Ozseker HS, Himmetoglu C et al. Omentectomy for gynecologic cancer: how much sampling is adequate for microscopic examination? Arch Pathol Lab Med 2007;131:1578-1581.

29 Seidman JD, Soslow RA, Vang $\mathrm{R}$ et al. Borderline ovarian tumors: diverse contemporary viewpoints on terminology and diagnostic criteria with illustrative images. Hum Pathol 2004;35:918-933.

30 Kurman RJ, Carcangiu ML, Herrington CS et al. WHO Classification of Tumours of the Female Reproductive Organs. IARC Press: Lyon, France, 2014.

31 McCluggage WG. My approach to and thoughts on typing of ovarian carcinomas. J Clin Pathol 2008;61: 152-163.

32 Shih IM, Kurman RJ. Ovarian tumorigenesis. A proposed model based on morphological and molecular genetic analysis. Am J Pathol 2004;164: 1511-1518.

33 Gilks CB. Subclassification of ovarian surface epithelial tumors based on correlation of histologic and molecular pathologic data. Int J Gynecol Pathol 2004;23:200-205.

34 Soslow RA. Histologic subtypes of ovarian carcinoma: an overview. Int J Gynecol Pathol 2008;27:161-174.

35 McCluggage WG. Malignant biphasic uterine tumours: carcinosarcomas or metaplastic carcinomas? J Clin Pathol 2002;55:321-325.

36 Downes MR, Allo G, McCluggage WG et al. Review of findings in prophylactic gynaecologic specimens in Lynch syndrome with literature review and recommendations for grossing. Histopathology 2014; 65:228-239.

37 Seidman JD, Horkayne-Szakaly I, Haiba M et al. The histologic type and stage distribution of ovarian carcinomas of surface epithelial origin. Int J Gynecol Pathol 2004;23:41-44.

38 Köbel M, Kalloger SE, Huntsman DG et al. Differences in tumor type in low-stage versus high-stage ovarian carcinomas. Int J Gynecol Pathol 2010;29:203-211. 
39 Chan JK, Tian C, Monk BJ et al. Prognostic factors for high-risk early-stage epithelial ovarian cancer: a Gynecologic Oncology Group study. Cancer 2008;112: 2202-2210.

40 Shimizu Y, Kamoi S, Amada S et al. Toward the development of a universal grading system for ovarian epithelial carcinoma: testing of a proposed system in a series of 461 patients with uniform treatment and follow-up. Cancer 1998;82:893-901.

41 Seidman JD, Horkayne-Szakaly I, Cosin JA et al. Testing of two binary grading systems for FIGO stage III serous carcinoma of the ovary and peritoneum. Gynecol Oncol 2006;103:703-708.

42 Malpica A, Deavers MT, Lu K et al. Grading ovarian serous carcinoma using a two-tier system. Am J Surg Pathol 2004;28:496-504.

43 Bodurka DC, Deavers MT, Tian C et al. Reclassification of serous ovarian carcinoma by a 2-tier system: a Gynecologic Oncology Group Study. Cancer 2012; 118:3087-3094.

44 Yamamoto S, Kasajima A, Takano M et al. Validation of the histologic grading for ovarian clear cell adenocarcinoma: a retrospective multi-institutional study by the Japan Clear Cell Carcinoma Study Group. Int J Gynecol Pathol 2011;30:129-138.

45 Zaino RJ, Kurgan RJ, Diana KL et al. The utility of the revised International Federation of Gynecology and Obstetrics histologic grading system. A Gynecologic Oncology Group Study. Cancer 1995;75:81-86.

46 Taylor RR, Zeller J, Lieberman RW et al. An analysis of two versus three grades for endometrial carcinoma. Gynecol Oncol 1999;92:119-123.

47 Takeshima N, Hirai Y, Hasumi K. Prognostic validity of neoplastic cells with notable nuclear atypia in endometrial cancer. Obstet Gynecol 1998;92: 119-123.

48 Lax SF, Kurgan RJ, Pizer ES et al. A binary architectural grading system for uterine endometrial endometrioid carcinoma has superior reproducibility compared with FIGO grading and identifies subsets of advance-stage tumors with favorable and unfavorable prognosis. Am J Surg Pathol 2000;24:1201-1208.

49 Scholten AN, Smit VT, Beerman H et al. Prognostic significance and interobserver variability of histologic grading system for endometrial carcinoma. Cancer 2004;100:764-772.

50 Alkushi A, Abdul-Rahman ZH, Lim P et al. Description of a novel system for grading of endometrial carcinoma and comparison with existing grading systems. Am J Surg Pathol 2005;29:295-304.

51 Bell DA, Longacre TA, Prat J et al. Serous borderline (low malignant potential, atypical proliferative) ovarian tumors: workshop perspectives. Hum Pathol 2004;35:934-948.

52 Seidman JD, Bell DA, Crum CP et al. Tumours of the ovary: epithelial tumours-serous tumours. In: Kurman RJ, Carcangiu ML, Herrington S et al. (eds). World Health Organization Classification of Tumours of Female Reproductive Organs, 4th (edn). IARC: Lyon, France, 2014.

53 Ellenson LH, Carinelli SG, Cho KR et al. Tumours of the ovary: epithelial tumours-endometrioid and endometrioid stromal tumours. In: Kurman RJ, Carcangiu ML, Herrington S et al. (eds). World Health Organization Classification of Tumours of Female Reproductive Organs, 4th (edn). IARC Press: Lyon, France, 2014.
54 Gilks CB, Bell DA, Huntsman D et al. Tumours of the ovary: epithelial tumours-clear cell tumours. In: Kurman RJ, Carcangiu ML, Herrington S et al. (eds). World Health Organization Classification of Tumours of Female Reproductive Organs, 4th (edn). IARC Press: Lyon, France, 2014.

55 Gilks CB, Carinelli SG, Lawrence WD et al. Tumours of the ovary: epithelial tumours-Brenner tumours. In: Kurman RJ, Carcangiu ML, Herrington $S$ et al. (eds). World Health Organization Classification of Tumours of Female Reproductive Organs, 4th (edn). IARC Press: Lyon, France, 2014.

56 Köbel M, Bell DA, Carcangiu ML et al. Tumours of the ovary: epithelial tumours-seromucinous tumours. In: Kurman RJ, Carcangiu ML, Herrington S et al. (eds). World Health Organization Classification of Tumours of Female Reproductive Organs, 4th (edn). IARC Press: Lyon, France, 2014.

57 Longacre T, Bell D, Malpica A et al. Tumours of the ovary: epithelial tumours-mucinous tumours. In: Kurman RJ, Carcangiu ML, Herrington S et al. (eds). World Health Organization Classification of Tumours of Female Reproductive Organs, 4th (edn). IARC Press: Lyon, France, 2014.

58 Ronnett BM, Kajdacsy-Balla A, Gilks CB et al. Mucinous borderline ovarian tumors: points of general agreement and persistent controversies regarding nomenclature, diagnostic criteria, and behavior. Hum Pathol 2004;35:949-960.

59 McKenney JK, Balzer BL, Longacre TA. Patterns of stromal invasion in ovarian serous tumors of low malignant potential (borderline tumors): a reevaluation of the concept of stromal microinvasion. Am J Surg Pathol 2006;30:1209-1221.

60 Bell DA, Weinstock MA, Scully RE. Peritoneal implants of ovarian serous borderline tumors. Histologic features and prognosis. Cancer 1988;62:2212-2222.

61 Bell KA, Smith Sehdev AE, Kurman RJ. Refined diagnostic criteria for implants associated with ovarian atypical proliferative serous tumors (borderline) and micropapillary serous carcinomas. Am J Surg Pathol 2001;25:419-432.

62 Longacre TA, McKenney JK, Tazelaar HD et al. Ovarian serous tumors of low malignant potential (borderline tumors): outcome-based study of 276 patients with long-term ( $>$ or $=5$-year) follow-up. Am J Surg Pathol 2005;29:707-723.

63 Ardighieri L, Zeppernick F, Hannibal CG et al. Mutational analysis of BRAF and KRAS in ovarian serous borderline (atypical proliferative) tumours and associated peritoneal implants. J Pathol 2014;232: 16-22.

64 Carlson JW, Jarboe EA, Kindelberger D et al. Serous tubal intraepithelial carcinoma: diagnostic reproducibility and its implications. Int J Gynecol Pathol 2010;29:310-314.

65 Visvanathan K, Vang R, Shaw P et al. Diagnosis of serous tubal intraepithelial carcinoma (STIC) based on morphologic and immunohistochemical features. A reproducibility study. Am J Surg Pathol 2011;35: 1766-1775.

66 Vang R, Visvanathan K, Gross A et al. Validation of an algorithm for the diagnosis of serous tubal intraepithelial carcinoma. Int J Gynecol Pathol 2012;31: 243-253.

67 Tang S, Onuma K, Deb P et al. Frequency of serous tubal intraepithelial carcinoma in various gynecologic 
malignancies: a study of 300 consecutive cases. Int J Gynecol Pathol 2012;31:103-110.

68 Jarboe EA, Miron A, Carlson JW et al. Coexisting intraepithelial serous carcinomas of the endometrium and fallopian tube: frequency and potential significance. Int J Gynecol Pathology 2009;28:308-315.

69 Rabban JT, Vohra P, Zaloudek C. Intramucosal growth in fallopian tube fimbriae by tumors of nongynecologic origin may mimic serous tubal intraepithelial carcinoma and tubal mucinous metaplasia. Mod Pathol 2014;27:302A.

70 McCluggage WG. WT1 is of value in ascertaining the site of origin of serous carcinomas within the female genital tract. Int J Gynecol Pathol 2004;23:97-99.

71 Berek JS. Lymph node-positive stage IIIC ovarian cancer. A separate entity? Int J Gynecol Cancer 2009;19:S18-S20.

72 Bakker R, Gershenson D, Fox P et al. Stage IIIC ovarian/ peritoneal serous carcinoma: a heterogenous group of patients with different prognosis. Int J Gynecol Cancer 2014;33:302-308.

73 Fadare O. Recent developments on the significance and pathogenesis of lymph node involvement in ovarian serous tumors of low malignant potential (borderline tumors). Int J Gynecol Cancer 2009;19: 103-108.

74 Morice P, Uzan C, Fauvet R et al. Borderline ovarian tumour: pathological diagnostic dilemma and risk factors for invasive or lethal recurrence. Lancet Oncol 2012;13:e103-e115.

75 McKenney JK, Balzer BL, Longacre TA. Lymph node involvement in ovarian serous tumors of low malignant potential (borderline tumors): pathology, prognosis, and proposed classification. Am J Surg Pathol 2006;30:614-624.

76 Djordjevic B, Malpica A. Ovarian serous tumors of low malignant potential with nodal low-grade serous carcinoma. Am J Surg Pathol 2012;36:955-963.

77 Hennessy BT, Coleman RL, Markman M. Ovarian cancer. Lancet 2009;374:1371-1382.

78 Siegel R, Ma J, Zou Z et al. Cancer statistics,2014. CA Cancer J Clin 2014;64:9-29.

79 Folkins AK, Longacre TA. Hereditary gynaecological malignancies: advances in screening and treatment. Histopathology 2013;62:2-30.

80 Alsop K, Fereday S, Meldrum C et al. BRCA mutation frequency and patterns of treatment response in BRCA mutation-positive women with ovarian cancer: a report from the Australian Ovarian Cancer Study Group. J Clin Oncol 2012;26:2654-2663.

81 Soslow RA, Han G, Park KJ et al. Morphologic patterns associated with BRCA1 and BRCA2 genotype in ovarian carcinoma. Mod Pathol 2012;25: $625-636$.

82 Fujiwara M, McGuire VA, Felberg A et al. Prediction of BRCA1 germline mutation status in women with ovarian cancer using morphology-based criteria. Identification of a BRCA1 ovarian cancer phenotype. Am J Surg Pathol 2012;36:1170-1177.

83 Dean E, El-Helw L, Hasan J. Targeted therapies in epithelial ovarian cancer. Cancers 2010;2:88-113.

84 Chui $\mathrm{MH}$, Gilks B, Cooper $\mathrm{K}$ et al. Identifying Lynch syndrome in patients with ovarian carcinoma: the significance of tumor type. Adv Anat Pathol 2013;20:378-386.

85 Singh N. Synchronous tumours of the female genital tract. Histopathology 2010;56:277-285.
86 Garg K, Soslow RA. Lynch syndrome (hereditary nonpolyposis colorectal cancer) and endometrial carcinoma. J Clin Pathol 2009;62:679-684.

87 Miettinen M. Overview of soft tissue tumors. In: Miettinen M(ed). Modern Soft Tissue Pathology: Tumors and Non-Neoplastic Conditions. Cambridge University Press: New York, NY, USA, 2010, pp 1-10.

88 Gramlich T, Austin RM, Lutz M. Histologic sampling requirements in ovarian carcinoma: a review of 51 tumors. Gynecol Oncol 1990;38:249-256.

89 Silverberg SG, Bell DA, Kurman RJ et al. Borderline ovarian tumors: key points and workshop summary. Hum Pathol 2004;35:910-917.

90 Seidman JD, Kurman RJ, Ronnett BM. Primary and metastatic mucinous adenocarcinomas in the ovaries: incidence in routine practice with a new approach to improve intraoperative diagnosis. Am J Surg Pathol 2003;27:985-993.

91 Khunamornpong S, Suprasert P, Pojchamarnwiputh S et al. Primary and metastatic mucinous adenocarcinomas of the ovary: Evaluation of the diagnostic approach using tumor size and laterality. Gynecol Oncol 2006;101:152-157.

92 Rodríguez IM, Prat J. Mucinous tumors of the ovary: a clinicopathologic analysis of 75 borderline tumors (of intestinal type) and carcinomas. Am J Surg Pathol 2002;26:139-152.

93 Lee KR, Scully RE. Mucinous tumors of the ovary: a clinicopathologic study of 196 borderline tumors (of intestinal type) and carcinomas, including an evaluation of 11 cases with 'pseudomyxoma peritonei'. Am J Surg Pathol 2000;24:1447-6.

94 Nomura K, Aizawa S. Noninvasive, microinvasive, and invasive mucinous carcinomas of the ovary: a clinicopathologic analysis of 40 cases. Cancer 2000;89:1541-1546.

95 Chen S, Leitao MM, Tornos C et al. Invasion patterns in stage $\mathrm{I}$ endometrioid and mucinous ovarian carcinomas: a clinicopathologic analysis emphasizing favorable outcomes in carcinomas without destructive stromal invasion and the occasional malignant course of carcinomas with limited destructive stromal invasion. Mod Pathol 2005;18:903-911.

96 Ludwick C, Gilks CB, Miller D et al. Aggressive behavior of stage I ovarian mucinous tumors lacking extensive infiltrative invasion: a report of four cases and review of the literature. Int J Gynecol Pathol 2005;24:205-217.

97 Tabrizi AD, Kalloger SE, Köbel M et al. Primary ovarian mucinous carcinoma of intestinal type: significance of pattern of invasion and immunohistochemical expression profile in a series of 31 cases. Int J Gynecol Pathol 2010;29:99-10.

98 Ariyoshi K, Kawauchi S, Kaku T et al. Prognostic factors in ovarian carcinosarcoma: a clinicopathological and immunohistochemical analysis of 23 cases. Histopathology 2000;37:427-436.

99 Rutledge TL, Gold MA, McMeekin DS et al. Carcinosarcoma of the ovary-a case series. Gynecol Oncol 2006;100:128-132.

$100 \mathrm{Lu} \mathrm{CH}$, Chen IH, Chen YJ et al. Primary treatment and prognostic factors of carcinosarcoma of the ovary, fallopian tube, and peritoneum: a Taiwanese Gynecologic Oncology Group Study. Int J Gynecol Cancer 2014;24:506-512.

101 Fisher B, Bryant J, Wolmark $\mathrm{N}$ et al. Effect of preoperative chemotherapy on the outcome of women 
with operable breast cancer. J Clin Oncol 1998;16: 2672-2685.

102 Rodel C, Martus P, Papadoupolos T et al. Prognostic significance of tumor regression after preoperative chemoradiotherapy for rectal cancer. J Clin Oncol 2005;23:8688-8696.

103 Ruo L, Tickoo S, Klimstra DS et al. Long-term prognostic significance of extent of rectal cancer response to preoperative radiation and chemotherapy. Ann Surg 2002;236:75-81.

104 Wolmark N, Wang J, Mamounas E et al. Preoperative chemotherapy in patients with operable breast cancer: nine-year results from National Surgical Adjuvant Breast and Bowel Project B-18. J Natl Cancer Inst Monogr 2001;30:96-102.

105 Gavioli M, Luppi G, Losi L et al. Incidence and clinical impact of sterilized disease and minimal residual disease after preoperative radiochemotherapy for rectal cancer. Dis Colon Rectum 2005;48:1851-1857.

106 Ogston KN, Miller ID, Payne S et al. A new histological grading system to assess response of breast cancers to primary chemotherapy: prognostic significance and survival. Breast 2003;12:320-327.

107 Sataloff DM, Mason BA, Prestipino AJ et al. Pathologic response to induction chemotherapy in locally advanced carcinoma of the breast: a determinant of outcome. J Am Coll Surg 1995;180:297-306.

108 Corben AD, Abi-Raad R, Popa I et al. Pathologic response and long-term follow-up in breast cancer patients treated with neoadjuvant chemotherapy: a comparison between classifications and their practical application. Arch Pathol Lab Med 2013;137: 1074-1082.

109 Mandard AM, Dalibard F, Mandard JC et al. Pathologic assessment of tumor regression after preoperative chemoradiotherapy of esophageal carcinoma. Clinicopathologic correlation. Cancer 1994;73:2680-2686.

110 Dworak O, Keilholz L, Hoffmann A. Pathological features of rectal cancer after preoperative radiochemotherapy. Int J Colorectal Dis 1997;12:19-23.

111 Chetty R, Gill P, Govender D et al. International study group on rectal cancer regression grading: interobserver variability with commonly used regression grading systems. Hum Pathol 2012;43:1917-1923.

112 Chetty R, Gill P, Bateman AC et al. Pathological grading of regression: an International Study Group perspective. J Clin Pathol 2012;65:865-866.

113 Chetty R, Gill P, Govender D et al. A multi-centre pathologist survey on pathological processing and regression grading of colorectal cancer resection specimens treated by neoadjuvant chemoradiation. Virchows Arch 2012;460:151-155.

114 Thies S, Langer R. Tumor Regression grading of gastrointestinal carcinomas after neoadjuvant treatment. Front Oncol 2013;3:262.

115 Le T, Williams K, Senterman M et al. Histopathologic assessment of chemotherapy effects in epithelial ovarian cancer patients treated with neoadjuvant chemotherapy and delayed primary surgical debulking. Gynecol Oncol 2007;106:160-163.

116 Muraji M, Sudo T, Iwasaki S et al. Histopathology predicts clinical outcome in advanced epithelial ovarian cancer patients treated with neoadjuvant chemotherapy and debulking surgery. Gynecol Oncol 2013;131:531-534.

117 Petrillo M, Zannoni GF, Tortorella L et al. Prognostic role and predictors of complete pathologic response to neoadjuvant chemotherapy in primary unresectable ovarian cancer. Am J Obstet Gynecol 2014;211, 632 e1-e8.

118 Sassen S, Schmalfeldt B, Avril N et al. Histopathologic assessment of tumor regression after neoadjuvant chemotherapy in advanced-stage ovarian cancer. Hum Pathol 2007;38:926-934.

119 Boehm S, Said I, Faruqi A et al. Development of a response scoring system to quantify the effect of neoadjuvant chemotherapy in ovarian cancerovarian cancer response scoring (OCRS) study. Mod Pathol 2014;27:276A.

120 McCluggage WG. Recent advances in immunohistochemistry in the diagnosis of ovarian neoplasms. J Clin Pathol 2000;53:558-560.

121 McCluggage WG. Recent advances in immunohistochemistry in gynaecological pathology. Histopathology 2002;46:309-326.

122 McCluggage WG, Young RH. Immunohistochemistry as a diagnostic aid in the evaluation of ovarian tumors. Semin Diagn Pathol 2005;22:3-32.

123 Shimizu M, Toki T, Takagi Y et al. Immunohistochemical detection of the Wilms' tumor gene (WT1) in epithelial ovarian tumors. Int J Gynecol Pathol 2000;19:158-163.

124 Al-Hussaini M, Stockman A, Foster H et al. WT-1 assists in distinguishing ovarian from uterine serous carcinoma and in distinguishing between serous and endometrioid ovarian carcinoma. Histopathology 2004;44:109-115.

125 Goldstein NS, Uzieblo A. WTI immunoreactivity in uterine papillary serous carcinomas is different from ovarian serous carcinomas. Am J Clin Pathol 2002; 117:541-545.

126 Acs G, Pasha T, Zhang PJ. WT1 is expressed in serous, but not in endometrioid, clear cell or mucinous carcinoma of the peritoneum, fallopian tube, ovaries and endometrium. Int J Gynecol Pathol 2004;23:110-118.

127 Hashi A, Yuminamochi T, Murata S et al. Wilms' tumor gene immunoreactivity in primary serous carcinomas of the fallopian tube, ovary, endometrium, and peritoneum. Int J Gynecol Pathol 2003;22:374-377.

128 Stewart CJ, Brennan BA, Chan T et al. WT1 expression in endometrioid ovarian carcinoma with and without associated endometriosis. Pathology 2008;40: 592-599.

129 O’Neill CJ, McBride HA, Connolly LE et al. High-grade ovarian serous carcinoma exhibits significantly higher p16 expression than low-grade serous carcinoma and serous borderline tumour. Histopathology 2007;50: 773-779.

130 DeLair D, Oliva E, Koble $\mathrm{M}$ et al. Morphologic spectrum of immunohistochemically characterized clear cell carcinoma of the ovary: a study of 155 cases. Am J Surg Pathol 2011;35:36-44.

131 Yamashita Y, Nagasaka T, Naiki-Ito A et al. Napsin A is a specific marker for ovarian clear cell adenocarcinoma. Mod Pathol 2015;28:111-117.

132 McCluggage WG, Wilkinson N. Metastatic neoplasms involving the ovary: a review with an emphasis on morphological and immunohistochemical features. Histopathology 2005;47:231-247.

133 Vang R, Gown AM, Barry TS et al. Cytokeratins 7 and 20 in primary and secondary mucinous tumors of the ovary: analysis of coordinate immunohistochemical expression profiles and staining distribution in 179 cases. Am J Surg Pathol 2006;30:1130-1139. 
134 Ji H, Isacson C, Seidman JD et al. Cytokeratins 7 and 20, Dpc4 and MUC5AC in the distinction of metastatic mucinous carcinomas in the ovary from primary ovarian mucinous carcinomas: Dpc4 assists in identifying metastatic pancreatic carcinomas. Int J Gynecol Pathol 2002;21:391-400.

135 Nonaka D, Chiriboga L, Soslow RA. Expression of pax8 as a useful marker in distinguishing ovarian carcinomas from mammary carcinomas. Am J Surg Pathol 2008;32:1566-1571.

136 Tornos C, Soslow R, Chen S et al. Expression of WT1, CA125, and GCDFP-15 as useful markers in the differential diagnosis of primary ovarian carcinomas versus metastatic breast cancer to the ovary. Am J Surg Pathol 2005;29:1482-1489.

137 Liu H, Shi J, Wilkerson ML et al. Immunohistochemical evaluation of GATA3 expression in tumors and normal tissues: a useful immunomarker for breast and urothelial carcinomas. Am J Clin Pathol 2012;138: 57-64.

138 Bhargava R, Beriwal S, Dabbs DJ. Mammaglobin vs GCDFP-15: an immunohistologic validation survey for sensitivity and specificity. Am J Clin Pathol 2007;127: 103-113.

139 Ronnett BM, Yemelyanova AV, Vang R et al. Endocervical adenocarcinomas with ovarian metastases: analysis of 29 cases with emphasis on minimally invasive cervical tumors and the ability of the metastases to simulate primary ovarian neoplasms. Am J Surg Pathol 2008;32:1835-1853.

140 McCluggage WG, Young RH. Ovarian sertoli-leydig cell tumors with pseudoendometrioid tubules (pseudoendometrioid sertoli-leydig cell tumors). Am J Surg Pathol 2007;31:592-597.

141 Zhao C, Barner R, Vinh TN et al. SF-1 is a diagnostically useful immunohistochemical marker and comparable to other sex cord-stromal tumor markers for the differential diagnosis of ovarian Sertoli cell tumor. Int J Gynecol Pathol 2008;27:507-514.

142 Zhao C, Vinh TN, McManus K et al. Identification of the most sensitive and robust immunohistochemical markers in different categories of ovarian sex cordstromal tumors. Am J Surg Pathol 2009;33:354-366.

143 Vang R, Shih IM, Kurman RJ. Fallopian tube precursors of ovarian low- and high-grade serous neoplasms. Histopathology 2013;62:44-58.

144 McCluggage WG, Connolly LE, McGregor G et al. A strategy for defining biologically relevant levels of p53 protein expression in clinical samples with reference to endometrial neoplasia. Int J Gynecol Pathol 2005;24:307-312.

145 Kobel M, Reuss A, Du Bois A et al. The biological and clinical value of p53 expression in pelvic high-grade serous carcinomas. J Pathol 2010;222:191-198.

146 McCluggage WG, Soslow RA, Gilks CB. Patterns of p53 immunoreactivity in endometrial carcinomas: 'all or nothing' staining is of importance. Histopathology 2011;59:786-788.

147 Zaino R, Whitney C, Brady MF et al. Simultaneously detected endometrial and ovarian carcinoma: a prospective clinicopathologic study of 74 cases: a Gynecologic Oncology Group Study. Gynecol Oncol 2001;83:355-362.

148 Ayhan A, Yalcin OT, Tuncer ZS et al. Synchronous primary malignancies of the female genital tract. Eur J Obstet Gynecol Reprod Biol 1992;45:63-66.

149 Hirschowitz L, Ganesan R, McCluggage WG. WT1, p53 and hormone receptor expression in uterine serous carcinoma. Histopathology 2009;55:478-482.

150 Oparka R, McCluggage WG, Herrington CS. Peritoneal mesothelial hyperplasia associated with gynaecological disease: a potential diagnostic pitfall that is commonly associated with endometriosis. J Clin Pathol 2011;64:313-318.

151 Prat J. Ovarian carcinomas: five distinct diseases with different origins, genetic alterations, and clinicopathological features. Virchows Arch 2012;460:237-249.

152 Kurman RJ, Shih I-M. The origin and pathogenesis of epithelial ovarian cancer: a proposed unifying theory. Am J Surg Pathol 2010;34:433-443.

153 The Cancer Genome Atlas Research Network. Integrated genomic analyses of ovarian carcinoma. Nature 2011;474:609-615.

154 Gilks CB. Molecular abnormalities in ovarian cancer subtypes other than high-grade serous carcinoma. J Oncol 2010;2010:740968.

155 Maeda D, Shih I-M. Pathogenesis and the role of ARID1A mutation in endometriosis-related ovarian neoplasms. Adv Anat Pathol 2013;20:45-52.

156 Cuatrecasas M, Villanueva A, Matias-Guiu X et al. Kras mutations in mucinous ovarian tumors. Cancer 1997;79:1581-1586.

157 Matias-Guiu X, Prat J. Molecular pathology of endometrial carcinoma. Histopathology 2013;62: 111-123.

158 Matsuo K, Sheridan TB, Yoshino K et al. Significance of lymphovascular space invasion in epithelial ovarian cancer. Cancer Med 2012;1:156-164.

159 Matsuo K, Yoshino K, Hiramatsu K et al. Effect of lymphovascular space invasion on survival of stage I epithelial ovarian cancer. Obstet Gynecol 2014;123: 957-965.

160 Qian X, Xi X, Jin Y. The grading of lymphovascular space invasion in epithelial ovarian carcinoma. Int J Gynecol Cancer 2010;20:895-899.

161 Alvarado-Cabrero I, Stolnicu S, Kiyokawa T et al. Carcinoma of the fallopian tube: results of a multiinstitutional retrospective analysis of 127 patients with evaluation of staging and prognostic factors. Ann Diagn Pathol 2013;17:159-164.

162 Alvarado-Cabrero I, Young RH, Vamvakas EC et al. Carcinoma of the fallopian tube: a clinicopathological study of 105 cases with observations on staging and prognostic factors. Gynecol Oncol 1999;72:367-379.

163 Asmussen M, Kaern J, Kjoerstad K et al. Primary adenocarcinoma localized to the fallopian tubes: report on 33 cases. Gynecol Oncol 1988;30:183-186.

164 Baekelandt M, Jorunn Nesbakken A, Kristensen GB et al. Carcinoma of the fallopian tube. Cancer 2000;89: 2076-2084. 